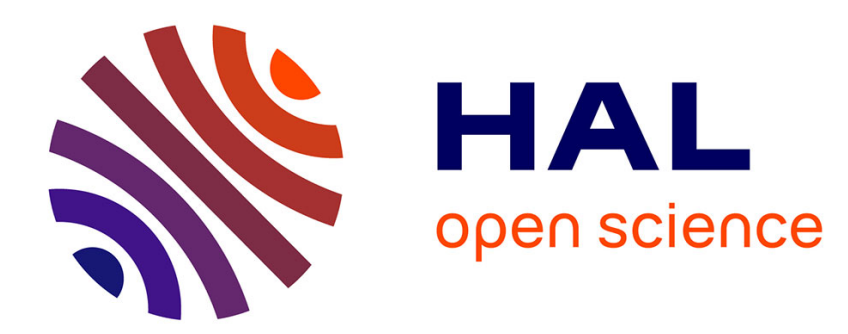

\title{
Monte-Carlo simulations of strongly interacting dipolar fluids between two conducting walls
}

\author{
Sabine H.L. Klapp
}

\section{To cite this version:}

Sabine H.L. Klapp. Monte-Carlo simulations of strongly interacting dipolar fluids between two conducting walls. Molecular Simulation, 2006, 32 (08), pp.609-621. 10.1080/08927020600883269 . hal00514995

\section{HAL Id: hal-00514995 \\ https://hal.science/hal-00514995}

Submitted on 4 Sep 2010

HAL is a multi-disciplinary open access archive for the deposit and dissemination of scientific research documents, whether they are published or not. The documents may come from teaching and research institutions in France or abroad, or from public or private research centers.
L'archive ouverte pluridisciplinaire HAL, est destinée au dépôt et à la diffusion de documents scientifiques de niveau recherche, publiés ou non, émanant des établissements d'enseignement et de recherche français ou étrangers, des laboratoires publics ou privés. 


\section{Molecular Simulation}

Journal of

Experimental Nanoscience

- Taylor \& Francis

Taylor \& Francis Group

\section{Monte-Carlo simulations of strongly interacting dipolar fluids between two conducting walls}

\begin{tabular}{|r|l|}
\hline Journal: & Molecular Simulation/Journal of Experimental Nanoscience \\
\hline Manuscript ID: & GMOS-2006-0033.R1 \\
\hline Journal: & Molecular Simulation \\
\hline Date Submitted by the \\
Author: & 22-Jun-2006 \\
\hline Complete List of Authors: & $\begin{array}{l}\text { Klapp, Sabine; Technical University Berlin, Stranski-Laboratorium, } \\
\text { Sekr. C7; Technical University Berlin, Institute of Theoretical } \\
\text { Physics, Sekr. PN 7-1 }\end{array}$ \\
\hline Keywords: & $\begin{array}{l}\text { computer simulations, Ewald summation, dipolar fluids, conducting } \\
\text { walls }\end{array}$ \\
\hline
\end{tabular}

\section{SCHOLARONE ${ }^{\text {M }}$ Manuscripts}




\title{
Monte-Carlo simulations of strongly interacting dipolar fluids between two conducting walls
}

\author{
Sabine H. L. Klapp*,†1 \\ 1* Stranski-Laboratorium für Physikalische und Theoretische Chemie, \\ Sekretariat TC7, Technische Universität Berlin, \\ Straße des 17. Juni 115, D-10623 Berlin, Germany \\ † Institut für Theoretische Physik, Sekretariat PN 7-1, \\ Fakultät II für Mathematik und Naturwissenschaften, \\ Technische Universität Berlin, Hardenbergstraße 36, D-10623 Berlin, Germany
}

(Dated: June 22, 2006)

\begin{abstract}
We report Monte-Carlo simulation results for a strongly interacting dipolar soft-sphere (DSS) fluid confined between two conducting, planar walls. The long-range dipolar interactions, including contributions from the "image dipoles" in the metal, are handled by mapping onto a problem with three-dimensional periodicity which can be treated by conventional Ewald summation methods. Considering two different wall separations our results indicate the occurence of wall-induced local and long-range ordering very similar to the related case of insulating walls. To understand this behavior we present ground-state (lattice) calculations on the basis of the Ewald sums, as well as simple macroscopic arguments appropriate for dipolar systems between conducting walls.

Keywords: Monte Carlo simulations, Ewald summation, dipolar fluids, phase behavior, confinement, conducting walls
\end{abstract}




\section{INTRODUCTION}

In recent years there has been substantial progress in simulating complex systems influenced by long-ranged, electrostatic interactions such as the Coulomb interactions between charged particles or the dipole-dipole interactions between particles with electric or magnetic dipole moments. Interactions of this type play an important and sometimes even dominant role in almost all biological systems, such as proteins, DNA, or charged membranes, and in electrolyte solutions. Moreover, is a wealth of technologically important substances such as polyelectrolytes [1] where electrostatic interactions determine the material properties. Other examples can be found among colloidal liquids [2] such as ferrofluids [3-5] (dispersions of ferromagnetic nanoparticles) and electrorheological fluids [6] (colloidal dispersions of polarizable particles).

In many applications involving such systems one is faced with some sort of spatial confinement. Examples range widely from cell membranes, electrolyte solutions near charged surfaces, proteins near (charged) membranes, polyelectrolyte films [7], phospholipid bilayers $[8,9]$, and thin films of magnetic colloids or ferrofluids [10]. This renders the question for the correct treatment of the long-range interactions in computer simulations of systems with reduced spatial symmetry. Within the last years much work has been done in generalizing and optimizing methods originally designed for three-dimensional Coulombic or dipolar systems, in particular the so-called Ewald summmation methods [11-14], to "simple" confined geometries such as slits (see e.g., Refs. [15-22]). Up to now most simulations studies for slitlike systems refer to fluids near or in between insulating walls, i.e. non-polarizable materials characterized by a dielectric constant $\epsilon_{\mathrm{W}}=1$ (or a magnetic permeability $\mu_{\mathrm{W}}=1$, respectively) [22-27]. However, particularly in the electric case one might also want to consider dielectric interfaces $\left(\epsilon_{\mathrm{W}}>1\right)$, which appear in many biological systems such as ion channels in proteins or self-assembled monolayers [29]. A special case in this context are conducting interfaces $\left(\epsilon_{\mathrm{W}}=\infty\right)$ which are particularly important in electrochemical problems [30] and in electrorheological fluids $[6,31]$. The present paper deals with the influence of conducting walls on a fluid of (spherical particles) with permanent dipole moments. This system may serve as a simple model of either a polar molecular fluid (that is, a solvent) or a dipolar colloidal fluid [2].

From a theoretical point of view, the crucial difference between a Coulombic or dipolar 
system subject to conducting walls and the case of insulating walls is that the charges or dipoles in the original slab-like system create "image charges" or "image dipoles", respectively, within the metal [32]. These images are, of course, merely theoretical constructs (rather than real charges or dipoles) which allow to solve the electrostatic boundary value problem stating that the electrostatic field must be perpendicular to the surface of the walls. Practically, the existence of the images implies that there are additional interactions to be taken into account such that treatment of liquids close to conducting walls seems even more complicated than the case $\epsilon_{\mathrm{W}}=1$. However, as has been demonstrated in Refs. [33, 34] for point charges, the energy of the system between conducting walls can be mapped onto a problem with three dimensional periodicity which can then be treated by conventional three-dimensional Ewald summation methods [13, 14].

The purpose of the present paper is, on one hand, to generalize the mapping procedure described in Ref. [33] to systems of permanent point dipoles. The second purpose is to explore the extent to which the wall boundary conditions $\left(\epsilon_{\mathrm{W}}=\infty\right.$ versus $\left.\epsilon_{\mathrm{W}}=1\right)$ influence the behavior of a confined dipolar model fluid with dominant dipolar interactions. To this end we perform Monte Carlo (MC) simulations and compare the results to previous MC data $[27,28]$ obtained by us for the same model fluid confined between insulating walls. Surprisingly, we find that the two systems behave very similar. In particular, both fluids display spontaneous polarization along a direction parallel to the walls at sufficiently large densities and wall separations. To understand these results we present both macroscopic arguments and, in addition, lattice calculations based on the Ewald method established in this paper.

The remainder of the paper is organized as follows. In Sec. II A we describe our model system, a dipolar soft sphere (DSS) fluid between smooth, structureless walls characterized by $\epsilon_{\mathrm{W}}=\infty$. An exact expression for the dipolar contribution to the energy, $U_{\mathrm{D}}$, of the system is derived in Sec. II B, where we also show that $U_{\mathrm{D}}$ can be easily calculated from that of a related system with three-dimensional periodicity. Details of the corresponding Ewald method and of our MC simulations, which are carried out in a constant-parallel-pressure ensemble, can be found in Secs. II C and II D, respectively. Numerical results are presented in Sec. III. We first consider (Sec. III A) systems with wall separation $L_{z} / \sigma=7.0$ which value has also been discussed in our previous study at $\epsilon_{\mathrm{W}}=1$ [27]. In the present work we additionally consider (Sec. III B) very narrow systems at $L_{\mathrm{z}} / \sigma=2.20$, where the particles 
are essentially confined to a monolayer. Finally, lattice calculations involving dipolar slabs with a face-centered-cubic positional structure and various types of perfect orientational order are presented in Sec. IV. Our conclusions are summarized in Sec. V.

\section{MODEL AND SIMULATION METHOD}

\section{A. Model system}

We consider a fluid composed of $N$ soft spheres with positions $\mathbf{r}_{i}$ and point dipole moments $\boldsymbol{\mu}_{i}, i=1, \ldots N$ at their centers. The pair potential of the resulting dipolar soft sphere (DSS) fluid is defined by

$$
u_{\mathrm{ff}}(i j)=u_{\mathrm{SR}}\left(r_{i j}\right)+\frac{\boldsymbol{\mu}_{i} \cdot \boldsymbol{\mu}_{j}}{r_{i j}^{3}}-3 \frac{\left(\boldsymbol{\mu}_{i} \cdot \mathbf{r}_{i j}\right)\left(\boldsymbol{\mu}_{j} \cdot \mathbf{r}_{i j}\right)}{r_{i j}^{5}},
$$

where the last two terms form the dipole-dipole potential, $r_{i j}=\left|\mathbf{r}_{i j}\right|=\left|\mathbf{r}_{i}-\mathbf{r}_{j}\right|$ is the particle separation, and

$$
u_{\mathrm{SR}}(r)=u_{\mathrm{SS}}(r)-u_{\mathrm{SS}}\left(r_{\mathrm{c}}\right)+\left.\left(r_{\mathrm{c}}-r\right) \frac{d u_{\mathrm{SS}}(r)}{d r}\right|_{r=r_{\mathrm{c}}}
$$

is the shifted-force version of the usual soft-sphere potential $u_{\mathrm{SS}}(r)=4 \epsilon_{0}(\sigma / r)^{12}$. Since the shifted-force potential and its first derivative go to zero continuously at $r=r_{\mathrm{c}}$ corrections due to the finite cutoff are not required. In the present calculations, $r_{\mathrm{c}}=2.5 \sigma$. Fluid molecules are confined by two planar solid walls separated by a distance $L_{\mathrm{z}}$ along the $z^{-}$ axis of the coordinate system and of infinite extent in the $x-y$ plane. Assuming that the interaction potential between fluid particles and atoms composing the wall is given by the soft-sphere potential $u_{\mathrm{SS}}(r)$, and averaging over the subspaces $z \leq-L_{\mathrm{z}} / 2$ and $z \geq L_{\mathrm{z}} / 2$ occupied by substrate particles, the fluid-wall potential follows as

$$
u_{\mathrm{FW}}^{(k)}(z)=\frac{4 \pi}{45} \epsilon_{0} \rho_{\mathrm{w}} \sigma^{3}\left(\frac{\sigma}{L_{\mathrm{z}} / 2 \pm z}\right)^{9},
$$

where the plus and the minus sign refer to the lower $(k=1)$ and upper $(k=2)$ wall, respectively. In Eq. (2.3), the reduced density of solid particles is set to $\rho_{\mathrm{w}} \sigma^{3}=1$.

\section{B. Energy of the dipolar system}

In this section we derive an exact expression for the energy of a dipolar many-particle system between two metallic walls, following closely previous work on systems of point 
charges $[33,34]$.

We start by considering one particle with dipole moment $\boldsymbol{\mu}_{i}=\left(\mu_{i, \mathrm{x}}, \mu_{i, \mathrm{y}}, \mu_{i, \mathrm{z}}\right)$ located at some position $\mathbf{r}_{i}$ in between the walls. To simplify notation, we assume here the walls to be located at $z=0$ and $z=L_{\mathrm{z}}$. According to the rules of electrostatics [32] which states that the tangential part of the electric field must vanish on conducting surfaces, the effect of one metallic wall, e.g. the upper wall at $z=L_{\mathrm{z}}$, consists in the creation of an "image dipole" within the upper wall. The position of the image is $\mathbf{r}_{i}^{\prime}=\left(2 L_{\mathrm{z}}-z_{i}\right) \hat{\mathbf{z}}=\mathbf{r}_{i}+2\left(L_{\mathrm{z}}-z_{i}\right)$ (with $\hat{\mathbf{z}}$ being the unit vector in $z$-direction), and its dipolar vector $\boldsymbol{\mu}_{i}^{\prime}$ is that of the original particle reflected at the $z$-axis (to see this, consider the dipole as an arrangement of two charges of opposite sign, each of which creates an image charge). Therefore,

$$
\boldsymbol{\mu}_{i}^{\prime}=\left(-\mu_{i, \mathrm{x}},-\mu_{i, \mathrm{y}}, \mu_{i, \mathrm{z}}\right)
$$

The total electrostatic field at a position $\mathbf{r}_{j} \neq \mathbf{r}_{i}$ between the walls is then given by the superposition of the fields arising from the original dipole, $\boldsymbol{\mu}_{i}$, and that of its image, $\boldsymbol{\mu}_{i}^{\prime}$.

Introduction of the second (lower) wall at $z=0$ changes the situation drastically since now not only the original particle, but also its upper image are mirrored in the lower wall. These images at $z<0$ induce in turn new images in the upper wall, and so forth, as illustrated in Fig. 1. For metallic walls of infinite thickness, each single dipole therefore generates an infinite number of images. The first group of images (which includes the one mentioned in the beginning) is characterized by reflected orientations $\boldsymbol{\mu}_{i}^{\prime}$ [see Eq. (2.4)] and positions

$$
\boldsymbol{r}_{i}^{\prime}=\boldsymbol{r}_{i}+2\left(n_{\mathrm{z}} L_{\mathrm{z}}-z_{i}\right) \hat{\mathbf{z}}, \quad n_{\mathrm{z}}=0, \pm 1, \pm 2, \ldots, \pm \infty
$$

The second group of images has the same orientation as the original particle, i.e. $\boldsymbol{\mu}_{i}^{\prime \prime}=\boldsymbol{\mu}_{i}$, and is characterized by positions

$$
\boldsymbol{r}_{i}^{\prime \prime}=\boldsymbol{r}_{i}+2 n_{\mathrm{z}} L_{\mathrm{z}} \hat{\mathbf{z}}, \quad n_{\mathrm{z}}= \pm 1, \pm 2, \ldots, \pm \infty
$$

Consider now a many-particle system of $N$ particles between the walls. The dipolar energy of this system can be written as

$$
\tilde{U}_{\mathrm{D}}=-\frac{1}{2} \sum_{i=1}^{N} \boldsymbol{\mu}_{i} \cdot\left(\mathbf{E}_{i}^{\mathrm{self}}+\mathbf{E}_{i}^{\mathrm{other}}\right),
$$

where $\mathbf{E}_{i}^{\text {self }}$ is the electrostatic field arising from the images of particle $i$, and $\mathbf{E}_{i}^{\text {other }}$ comprises the fields from the other particles $j \neq i$ and the fields from their images. Introducing the 
short-hand notation

$$
\boldsymbol{e}_{i}\left(\boldsymbol{\mu}_{j}, \boldsymbol{r}_{j}\right)=\frac{3 \boldsymbol{r}_{i j}\left(\boldsymbol{\mu}_{j} \cdot \boldsymbol{r}_{i j}\right)}{r_{i j}^{5}}-\frac{\boldsymbol{\mu}_{j}}{r_{i j}^{3}}
$$

the field $\mathbf{E}_{i}^{\text {self }}$ becomes

$$
\mathbf{E}_{i}^{\text {self }}=\sum_{n_{\mathrm{z}}=-\infty, n_{\mathrm{z}} \neq 0}^{\infty} \boldsymbol{e}_{i}\left(\boldsymbol{\mu}_{i}, \boldsymbol{r}_{i}+2 n_{\mathrm{z}} L_{\mathrm{z}} \hat{\mathbf{z}}\right)+\sum_{n_{\mathrm{z}}=-\infty}^{\infty} \boldsymbol{e}_{i}\left(\boldsymbol{\mu}_{i}^{\prime}, \boldsymbol{r}_{i}+2\left(n_{\mathrm{z}} L_{\mathrm{z}}-z_{i}\right) \hat{\mathbf{z}}\right),
$$

where the first (second) sum include contributions from images of $i$ with the same (reflected) orientation. Note that in the first sum, the term $n_{\mathrm{z}}=0$ (which corresponds to a position between the walls) is omitted while the second sum is unrestricted. Similarly, the field $\mathbf{E}_{i}^{\text {other }}$ is given by

$$
\begin{aligned}
\mathbf{E}_{i}^{\text {other }}= & \sum_{j=1, j \neq i}^{N}\left[\boldsymbol{e}_{i}\left(\boldsymbol{\mu}_{j}, \boldsymbol{r}_{j}\right)+\sum_{n_{\mathrm{z}}=-\infty, n_{\mathrm{z}} \neq 0}^{\infty} \boldsymbol{e}_{i}\left(\boldsymbol{\mu}_{j}, \boldsymbol{r}_{j}+2 n_{\mathrm{z}} L_{\mathrm{z}} \hat{\mathbf{z}}\right)\right. \\
& \left.+\sum_{n_{\mathrm{z}}=-\infty}^{\infty} \boldsymbol{e}_{i}\left(\boldsymbol{\mu}_{j}^{\prime}, \boldsymbol{r}_{j}+2\left(n_{\mathrm{z}} L_{\mathrm{z}}-z_{i}\right) \hat{\mathbf{z}}\right)\right]
\end{aligned}
$$

where the first sum includes contributions from the true neighbors of particle $i$ and the other two sums contain the corresponding image contributions. Inserting expressions (2.9) and (2.10) into Eq. (2.7), using Eq. (2.8), and summarizing we obtain

$$
\begin{aligned}
\tilde{U}_{\mathrm{D}}= & \frac{1}{2} \sum_{i=1}^{N} \sum_{j=1}^{N}\left\{\sum _ { n _ { \mathrm { z } } = - \infty } ^ { \infty } { } ^ { \prime } \left[\frac{\boldsymbol{\mu}_{i} \cdot \boldsymbol{\mu}_{j}}{\left|\boldsymbol{r}_{i j}+2 n_{\mathrm{z}} L_{\mathrm{z}} \hat{\mathbf{z}}\right|^{3}}\right.\right. \\
& \left.-3 \frac{\left(\boldsymbol{\mu}_{i} \cdot\left(\boldsymbol{r}_{i j}+2 n_{\mathrm{z}} L_{\mathrm{z}} \hat{\mathbf{z}}\right)\right)\left(\boldsymbol{\mu}_{j} \cdot\left(\boldsymbol{r}_{i j}+2 n_{\mathrm{z}} L_{\mathrm{z}} \hat{\mathbf{z}}\right)\right)}{\left|\boldsymbol{r}_{i j}+2 n_{\mathrm{z}} L_{\mathrm{z}} \hat{\mathbf{z}}\right|^{5}}\right] \\
& +\sum_{n_{\mathrm{z}}=-\infty}^{\infty}\left[\frac{\boldsymbol{\mu}_{i} \cdot \boldsymbol{\mu}_{j}^{\prime}}{\left|\boldsymbol{r}_{i j}+2 n_{\mathrm{z}} L_{\mathrm{z}} \hat{\mathbf{z}}+2 z_{j} \hat{\mathbf{z}}\right|^{3}}\right. \\
& \left.\left.-3 \frac{\left(\boldsymbol{\mu}_{i} \cdot\left(\boldsymbol{r}_{i j}+2 n_{\mathrm{z}} L_{\mathrm{z}} \hat{\mathbf{z}}+2 z_{j} \hat{\mathbf{z}}\right)\right)\left(\boldsymbol{\mu}_{j}^{\prime} \cdot\left(\boldsymbol{r}_{i j}+2 n_{\mathrm{z}} L_{\mathrm{z}} \hat{\mathbf{z}}+2 z_{j} \hat{\mathbf{z}}\right)\right)}{\left|\boldsymbol{r}_{i j}+2 n_{\mathrm{z}} L_{\mathrm{z}} \hat{\mathbf{z}}+2 z_{j} \hat{\mathbf{z}}\right|^{5}}\right]\right\},
\end{aligned}
$$

where the prime at the first sum over $n_{\mathrm{z}}$ indicates that the term $n_{\mathrm{z}}=0$ is omitted (only) for $i=j$. Furthermore, in writing the summands in Eq. (2.11) we have used that summation over $n_{\mathrm{z}}$ is equivalent to a summation over $-n_{\mathrm{z}}$.

Finally, the central cell comprising the $N$ original particles is repeated periodically along the $x$ - and $y$ - directions of the coordinate system, resulting in an infinitely extended slab of fluid confined between conducting walls. The corresponding total dipolar energy, $U_{\mathrm{D}}$, of this 


\section{Ewald sum}

To calculate the energy of the extended system we can employ the conventional Ewald sum for a three-dimensional system with conducting boundaries [11-14],

$$
\begin{aligned}
U_{\mathrm{D}}^{3 \mathrm{~d}, \mathrm{ex}}=2 U_{\mathrm{D}}= & \frac{1}{2} \sum_{i=1}^{2 N} \sum_{j \neq i}\left[\left(\boldsymbol{\mu}_{i} \cdot \boldsymbol{\mu}_{j}\right) B\left(r_{i j}, \alpha\right)\right. \\
& \left.-\left(\boldsymbol{\mu}_{i} \cdot \mathbf{r}_{i j}\right)\left(\boldsymbol{\mu}_{j} \cdot \mathbf{r}_{i j}\right) C\left(r_{i j}, \alpha\right)\right] \\
& +\frac{1}{2 V_{\mathrm{cell}}^{\mathrm{ex}}} \sum_{\mathbf{k} \neq \mathbf{0}} \frac{4 \pi}{k^{2}} \exp \left(-\frac{k^{2}}{4 \alpha^{2}}\right) \widetilde{M}(\mathbf{k}) \widetilde{M}^{*}(\mathbf{k}) \\
& -\frac{2 \alpha^{3}}{3 \sqrt{\pi}}(2 N) \mu^{2}
\end{aligned}
$$

The appearance of the lattice sums in Eq. (2.13) already indicates that the dipolar system between metallic walls has, in a way, three-dimensional (3d) periodicity. The basic cell of this three-dimensional array contains the original cell with the $N$ particles plus the first set of images, e.g., the $N$ images resulting from the presence of the lower wall alone. In fact, as we show explicitely in Appendix A, the energy of the extended system with altogether $2 \mathrm{~N}$

$$
U_{\mathrm{D}}=\frac{1}{2} U_{\mathrm{D}}^{3 \mathrm{~d}, \mathrm{ex}}
$$

Thus, the energy of a system with conducting interfaces can be conveniently calculated from the energy of the corresponding extended system. The latter is twice as large as the original system, but has the great advantage of having three-dimensional periodicity. 
In Eq. (2.15), the real space contribution has been formulated under the assumption that the convergence parameter $\alpha$ is large enough to consider only interactions within the central cell. This is satisfied with the present choice $\alpha / L=7.0$. Furthermore, the functions $B$ and $C$ are defined by $[13]$

$$
\begin{aligned}
& B(r, \alpha) \equiv\left[\frac{2 \alpha r}{\sqrt{\pi}} \exp \left(-\alpha^{2} r^{2}\right)+\operatorname{erfc}(\alpha r)\right] / r^{3} \\
& C(r, \alpha) \equiv\left[\frac{2 \alpha r}{\sqrt{\pi}}\left(3+2 \alpha^{2} r^{2}\right) \exp \left(-\alpha^{2} r^{2}\right)+3 \operatorname{erfc}(\alpha r)\right] / r^{5}
\end{aligned}
$$

where erfc is the complementary error function. We evaluate the real-space part by employing periodic boundary conditions in the $x$ - and $y$-directions and the minimum-image convention. As to the reciprocal part in Eq. (2.15), $V_{\text {cell }}^{\mathrm{ex}}=A\left(2 L_{\mathrm{z}}\right)=2 L^{2} L_{\mathrm{z}}$ is the volume of the extended simulation cell (assuming that the area $A=L_{\mathrm{x}} L_{\mathrm{y}}$ is a square, that is $\left.L_{\mathrm{x}}=L_{\mathrm{y}}=L\right)$. Furthermore,

$$
\widetilde{M}(\mathbf{k})=\sum_{i=1}^{2 N} \boldsymbol{\mu}_{i} \cdot \mathbf{k} \exp \left(i \mathbf{k} \cdot \boldsymbol{r}_{i}\right),
$$

and $\widetilde{M}^{*}(\mathbf{k})$ denotes its complex conjugate. Also, the reciprocal vectors of the (simpletetragonal) superlattice are given by $\mathbf{k}=\left(2 \pi m_{\mathrm{x}} / L, 2 \pi m_{\mathrm{y}} / L, 2 \pi m_{\mathrm{z}} /\left(2 L_{\mathrm{z}}\right)\right.$ where $m_{\mathrm{x}}, m_{\mathrm{y}}$, and $m_{\mathrm{z}}$ are integers. In the present study the reciprocal sum includes $\mathbf{k}$-vectors with length up to $m_{x}^{2}+m_{y}^{2}+m_{z}^{2}=80$.

\section{Monte Carlo method}

Simulations were performed in the constant-parallel-pressure ensemble, that is at fixed $\left(N, L_{\mathrm{z}}, P_{\|}, T\right)$ where $T$ is the temperature and $P_{\|}$is the transverse pressure exerted on the confined fluid in directions parallel to the walls. For given parallel pressure, the average area $\langle A\rangle$ (or lateral length $\langle L\rangle$ ) adjusts itself accordingly.

The simulations were carried out with $N=500$ particles. Each MC cycle consisted of an attempted translation or rotation of the $N$ particles, followed by an attempt to change the box area $A=L^{2}$ parallel to the walls. Employing Metropolis algorithm [14], the translation (rotation) of a particle, say $i$, is accepted with probability $p_{\text {trans } / \text { rot }}=\min \left\{1, \exp \left(-\beta \Delta U_{\mathrm{T}}(i)\right)\right\}$, where $\beta=1 / k_{B} T, k_{B}$ is Boltzmann's constant, and $\Delta U_{\mathrm{T}}(i)$ is the associated change in total 
configurational energy,

$$
U_{\mathrm{T}}=\sum_{i>j} u_{\mathrm{SR}}(i j)+\frac{1}{2} U_{\mathrm{D}}^{3 \mathrm{~d}, \mathrm{ex}}+\sum_{k=1}^{2} \sum_{i} u_{\mathrm{FW}}^{(k)}(i) .
$$

Moreover, changes of the box area from $A=L^{2}$ to $A^{\prime}=\left(L^{\prime}\right)^{2}$ are accepted on the basis of a modified Metropolis criterion involving the probability

$$
p=\min \left\{1, \exp \left(-\beta \Delta_{A \rightarrow A^{\prime}}\right)\right\},
$$

where the argument of the pseudo Boltzmann factor is given by [35]

$$
-\beta \Delta_{A \rightarrow A^{\prime}}=-\beta \Delta U_{\mathrm{T}}^{A \rightarrow A^{\prime}}-\beta P_{\|}\left(A-A^{\prime}\right) L_{\mathrm{z}}+N \ln \left(\frac{A}{A^{\prime}}\right) .
$$

The displacement parameter governing the changes in $A$ was adjusted such that 40-50 percent of the attempts were accepted. Finally, all simulations were started with randomly oriented particles located on a face-centered cubic lattice. A typical run then consisted of 120000-200000 cycles for equilibration, followed by a production period of about 80000150000 cycles.

\section{MONTE CARLO SIMULATION RESULTS}

Thermal properties of DSS fluids can be characterized by the reduced temperature $T^{*}=$ $k_{\mathrm{B}} T / \epsilon_{0}$ and the reduced dipole moment $m^{*}=\mu / \sqrt{\epsilon_{0} \sigma^{3}}$. In the present study, all simulations were performed at $T^{*}=1.35$ and $m^{*}=3.0$, corresponding to a strongly interacting fluid with dipolar coupling strength $\mu^{2} / k_{\mathrm{B}} T \sigma^{3}=m^{* 2} / T^{*} \approx 6.6$. These parameters have already been employed by us in previous MC simulations of DSS fluids confined by insulating walls $\left(\epsilon_{\mathrm{W}}=\right.$ 1) $[27,28]$ and in bulk DSS (Molecular Dynamics) simulations [36, 37]. In the following we first discuss (Sec. III A) simulation results for DSS between conducting walls $\left(\epsilon_{\mathrm{W}}=\infty\right)$ with reduced wall separation $L_{\mathrm{z}}^{*}=L_{\mathrm{z}} / \sigma=7.0$, which value has also been considered in Refs. $[27,28]$. We then turn in Sec. III B to an even narrower system characterized by $L_{\mathrm{z}}^{*}=2.2$.

\section{A. Wall separation $L_{\mathbf{z}}^{*}=7.0$}

Recently it was shown that dense bulk systems of DSS [36, 37] (and dipolar hard spheres $[38,39])$ may develop spontaneous polarization, yielding a liquid state with long-range 
ferroelectric order. In Ref. [27] we have demonstrated that this phase transition appears also in the presence of insulating walls with sufficiently large wall separation such as, e.g., $L_{\mathrm{z}}^{*}=7.0$. In fact, we found that those walls promote rather than suppress the long-range ordering: the transition occurs at somewhat lower pressures (and resulting average densities) compared to corresponding bulk systems.

Against this background it is interesting to see whether the present system characterized by $\epsilon_{\mathrm{W}}=\infty$ also polarizes spontaneously. To this end, we first consider the behavior of the global (i.e., pore-averaged) order parameters $P_{1}$ and $P_{2}$ [13] measuring the degree of polarization and the alignment, respectively. For a given configuration $P_{2}$ was taken to be the largest eigenvalue of the ordering matrix $\mathbf{Q}=(1 / N) \sum_{i=1}^{N}\left(3 \hat{\boldsymbol{\mu}}_{i} \hat{\boldsymbol{\mu}}_{i}-\mathbf{I}\right) / 2$ where $\hat{\boldsymbol{\mu}}_{i}$ is the unit vector associated with $\boldsymbol{\mu}_{i}$, and $\mathbf{I}$ is the identity matrix. The corresponding normalized eigenvector is the global director $\hat{\mathbf{d}}$, from which the instantaneous order parameter $P_{1}$ follows as

$$
P_{1}=\frac{1}{N}\left|\sum_{i=1}^{N} \hat{\boldsymbol{\mu}}_{i} \cdot \hat{\mathbf{d}}\right| .
$$

Numerical results for the average order parameters $\left\langle P_{1}\right\rangle$ and $\left\langle P_{2}\right\rangle$ as functions of the (externally applied) parallel pressure $P_{\|}^{*}=P_{\|} \sigma^{3} / \epsilon_{0}$ are plotted in Fig. 2 where we have included previous MC data (again with $N=500$ ) for the same DSS fluid but with insulating walls [27]. Starting from $P_{\|}^{*}=0.05$ and increasing the pressure up to $P_{\|}^{*} \approx 2.0$, the order parameters of the present system with conducting walls remains at first very small indicating that the system is globally isotropic in this pressure range (the small non-zero values of the order parameters can be attributed to finite-size effects [40]). Upon further compression, however, $\left\langle P_{1}\right\rangle$ and $\left\langle P_{2}\right\rangle$ sharply increase and for pressures $P_{\|}^{*}>2.8$ one observes very large order parameters with values $\left\langle P_{1}\right\rangle \geq 0.7$ and $\left\langle P_{2}\right\rangle \geq 0.5$ (note that the maximum value of these quantities is 1). Keeping in mind that all simulations were started from randomly oriented states, we conclude that these high-pressure states are indeed spontaneously polarized and therefore correspond to a true ferroelectric phase. Moreover, all states appearing in Fig. 2 are still fluid-like in directions parallel to the walls, as we have verified by an inspection of the in-plane correlation functions.

Comparing then the results obtained at $\epsilon_{\mathrm{W}}=\infty$ with those at $\epsilon_{\mathrm{W}}=1$ one finds that the pressure range in which the ferroelectric order develops upon compressing the fluid from the dilute limit, is essentially indistinguishable for the two wall boundary conditions 


$$
\mathbf{E}^{\mathrm{DP}}=-4 \pi D(k) \mathbf{P},
$$

where $D(k)$ is the depolarizing factor. The infinitely extended fluid slab in which we are interested here, is a special case of the ellipsoid where $k \rightarrow 0$, implying $D(k \rightarrow 0)=1[41]$. Assuming polarization normal to the insulating walls (i.e., $\epsilon_{\mathrm{W}}=\epsilon^{\prime}=1$ ) the fluid slab then experiences the field $\mathbf{E}^{\mathrm{DP}}=-4 \pi \mathbf{P}$ from which the corresponding energy per volume follows as $U^{\mathrm{DP}} / V=-(1 / 2) \mathbf{P} \cdot \mathbf{E}^{\mathrm{DP}}=2 \pi \mathbf{P}^{2}>0$. The apparance of this energy "penalty" explains why the DSS fluids simulated in Ref. [27], where $\epsilon_{\mathrm{W}}=1$, ordered along (arbitrary) directions parallel to the walls: for this situation, $D(k)=0$, and the depolarizing field vanishes [41].

Consider now the general ellipsoid immersed into a polarizable medium characterized by a dielectric constant $\epsilon^{\prime}>1$. In this case, the field $\mathbf{E}^{\mathrm{DP}}$ [see Eq. (3.2)] is supplemented by the so-called reaction field [42],

$$
\mathbf{E}^{\mathrm{RF}}=-4 \pi D(k) \frac{\left(\epsilon^{\prime}-1\right)(1-D(k))}{\left(\epsilon^{\prime}-1\right) D(k)-\epsilon^{\prime}} \mathbf{P} .
$$

Applying the formula (3.3) to a slab-like system $(D(k)=1)$ we see that the reaction field vanishes for this specific geometry regardless of the value of the $\epsilon^{\prime}=\epsilon_{\mathrm{W}}$ (contrary to, e.g, spherical samples where $D(k)=1 / 3$ and the reaction field cancels the depolarizing field in the limit $\left.\epsilon^{\prime} \rightarrow \infty\right)$. As a consequence, polarization normal to the walls is unfavorable even in presence of conducting walls $\left(\epsilon_{\mathrm{W}} \rightarrow \infty\right)$, at least from the perspective of macroscopic electrostatics. Indeed, further reasoning for the observed parallel ordering in our MC 
simulations is provided by the lattice calculations presented in Sec. IV.

In view of the macroscopic arguments above it is interesting to investigate the behavior of the total configurational potential energy [see Eq. (2.18)], $U_{\mathrm{T}}^{*}=U_{\mathrm{T}} / \epsilon_{0} N$, as obtained from the MC simulations. Results for $U_{\mathrm{T}}^{*}$ as function of $P_{\|}^{*}$ are plotted in Fig. 4. Upon increasing $P_{\|}$from zero the energy first rises in both systems as a consequence of the increasing repulsion [stemming from the short-range interactions, see Eq. (2.2)] between the particles. In this pressure range, the numerical values of $U_{\mathrm{T}}^{*}$ for metallic walls, on one hand, and insulating walls, on the other hand, are indeed essentially indistinguishable. Further compression then yields a sharp decrease of the total energy which can be attributed to the sudden decrease of dipolar energy (see inset in Fig. 4) associated to the onset of orientational ordering (see Fig. 2). Within the ferroelectric phase the energies then increase again upon increasing $P_{\|}^{*}$, with the values of $U_{\mathrm{T}}^{*}$ at high pressures being somewhat larger in the metallic case. An overview of thermodynamic data obtained at two examplary densities corresponding to the isotropic and ferroelectric phase is given in Tables I and II, respectively. The tables also include numerical estimates for the diagonal components of the (internal) pressure tensor (the off-diagonal elements should be zero in fluid states). Explicit expressions for the dipolar contribution to these quantities are given in Appendix B. Due to the planar, homogeneous character of the confining substrates, one expects the quantities $\tilde{P}_{\mathrm{xx}}$ and $\tilde{P}_{\mathrm{yy}}$ to agree with each other, and their average $\tilde{P}_{\|}=\left(\tilde{P}_{\mathrm{T}, \mathrm{xx}}+\tilde{P}_{\mathrm{T}, \mathrm{yy}}\right) / 2$ to coincide with the fixed input value $P_{\|}$ appearing in the Metropolis criterion [see Eq. (2.20)]. The data presented in Tables I and II indicate that these criteria are satisfied quite well even in the orientationally ordered phase. One also notes that the normal pressure, $P_{\mathrm{T}, \mathrm{zz}}^{*}$, is different from the parallel components, which is a direct consequence of the slab geometry.

Given the ordering behavior discussed so far it is not surprising that the local structure of the fluid confined between conducting walls resembles closely that between insulating walls. This may be verified from the local densities $\rho(z)=\langle N(z) /(A \Delta z\rangle$ ) (where $\Delta z=0.05 \sigma$ is the thickness of a thin slice centered on $z$ ) plotted in Fig. 5. Parts (a) and (b) correspond to the isotropic (a) and the ferroelectric (b) phase, respectively. As a consequence of the nanoscopic pore width the local density exhibits oscillations throughout the pore already at the relatively small pressure $P_{\|}^{*}=1.0$, but particularly at the larger pressure $P_{\|}^{*}=5.0$ related to the ferroelectric phase. These density oscillations indicate stratification of the fluid into layers of particles along directions parallel to the walls. In particular, the appearance 
of six layers at large pressures [see Fig. 5 (b)] is consistent with present value of the wall separation, $L_{\mathrm{z}}^{*}=7.0$. From a quantitative point of view, inspection of Fig. 5 shows that the local density is indeed essentially uneffected by the wall boundary conditions, the only difference being that the density peaks are somewhat more pronounced for $\epsilon_{\mathrm{W}}=1$ than for conducting walls.

Finally, to investigate the degree of local orientational order within the isotropic and ferroelectric phase we have calculated the order parameter [27]

$$
Q_{\mathrm{zz}}(z)=\left\langle\frac{\sum_{z}^{\prime}\left(3 \hat{\mu}_{i, \mathrm{z}}^{2}-1\right) / 2}{N(z)}\right\rangle,
$$

where the sum is carried out over particles within thin layers of thickness $\Delta z$. By definition, $Q_{\mathrm{zz}}(z)$ is zero in (globally isotropic, bulk-like) situations where the dipole moments rotate freely in all three spatial directions, whereas $Q_{\mathrm{zz}}(z)=-1 / 2$ when the dipoles point exclusively along directions in the $x-y$-plane. Note that such behavior does not imply any long-range orientational ordering but rather a restriction of dipole fluctuations normal to the walls. Therefore, $Q_{\mathrm{zz}}(z)$ can be non-zero even in a globally isotropic state. Numerical results for $Q_{\mathrm{zz}}(z)$ are given in Fig. 6 (a). Considering first the data corresponding to the isotropic phase $\left(P_{\|}^{*}=1.0\right)$ we see that $Q_{\mathrm{zz}}(z)$ is indeed negative particularly in the contact layers as a consequence of the presence of confining walls. Moreover, the present results are again very close to those obtained with insulating walls [27]. The same is true within the ferroelectric phase $\left(P_{\|}^{*}=5.0\right)$, where $Q_{\mathrm{zz}}(z)$ exhibits large negative values throughout the pore due to the long-range polarization parallel to the walls.

Finally, the local degree of polarization along the (pore-averaged) director $\hat{\mathbf{d}}$ of the ferroelectric phase can be detected from the order parameter

$$
P_{1}(z)=\left\langle\frac{\sum_{z}{ }^{\prime} \hat{\boldsymbol{\mu}}_{i} \cdot \hat{\mathbf{d}}}{N(z)}\right\rangle .
$$

Results are plotted in Fig. 6 (b), showing that indeed all layers of the confined ferroelectric fluid are polarized along the global director irrespective of the wall boundary conditions.

\section{B. Wall separation $L_{\mathrm{z}}^{*}=2.2$}

In view of our $\mathrm{MC}$ results at $L_{\mathrm{z}}^{*}=7.0$ it is interesting to see whether the wall boundary conditions have a similarly small effect when the spatial confinement becomes even more 
severe. To this end we have performed additional $\left(N, L_{\mathrm{z}}, P_{\|}, T\right) \mathrm{MC}$ simulations at $L_{\mathrm{z}}^{*}=2.2$. At this small pore width the particles essentially form monolayers regardless of the values of $P_{\|}^{*}$, as may be verified from plots of the local density $\rho(z)$ presented in Fig. 7 . Inspecting the density profiles in more detail one also realizes that they are fairly insensitive against $\epsilon_{\mathrm{W}}$ (the data presented here for insulating walls stem from $\left(N, L_{\mathrm{z}}, P_{\|}, T\right) \mathrm{MC}$ simulations with the slab-adapted Ewald method described in Ref. [27]). This is consistent with our findings at $L_{\mathrm{z}}^{*}=7.0$ (see Fig. 3).

However, comparing the two wall separations with each other it turns out that the confined fluids at $L_{\mathrm{z}}^{*}=2.2$ behave quite differently from the thicker films discussed in Sec. III A. In particular, the quasi-monolayer does not develop spontaneous polarization, i.e., the order parameter $\left\langle P_{1}\right\rangle$ remains very small within the pressure range $P_{\|}^{*} \leq 3.0$ investigated (at larger pressures, equilibration of the systems became increasingly difficult). This may be veryfied from plots of the orientational order parameters in Fig. 8. Recalling that the fluid at $L_{\mathrm{z}}^{*}=7.0$ orders at pressures $P_{\|}^{*} \approx 2.20$ (see Fig. 2 ) we conclude that the quasi-monolayer does not exhibit an isotropic-to-ferroelectric transition, at least not at comparable pressures. This holds for both wall boundary conditions considered. Interestingly, however, the systems at $L_{\mathrm{z}}^{*}=2.20$ were generally characterized by quite large degrees of average alignment parallel to the walls, as measured by the order parameter $\left\langle P_{2}\right\rangle$ also plotted in Fig. 8. Indeed, from this figure it is seen that the particles align relative to each other already at very small values of $P_{\|}^{*}$, with $\left\langle P_{2}\right\rangle$ being generally somewhat larger for conducting than for insulating walls. Two typical particle configurations ("snapshots") of the fluid particles are shown in Fig. 9. At very low pressures such as $P_{\|}^{*}=0.005$ [see Fig. 9 (a)], which corresponds to an average adsorption rate of $\Gamma^{*}=N \sigma^{2} /\langle A\rangle \approx 0.04$, the particles clearly arrange into large clusters and chains with head-to-tail alignment of the dipole moments. This is the typical behavior expected in strongly coupled dipolar fluids under dilute conditions [43]. In particular, the present "quasi-monolayers" behave very similar to true two-dimensional dipolar fluids [10] in that the chains form exclusively along "in-plane" directions, i.e., within the $x-y$ plane. At the larger pressure $P_{\|}^{*}=1.0\left(\Gamma^{*} \approx 0.71\right)$, the particles are still connected to chains but due to the larger density, the chains appear to align along a global director. There is, however, no sign of global parallel (ferroelectric) ordering, in accordance to the neglible values of $\left\langle P_{1}\right\rangle$ (see Fig. 8).

Finally, we compare in Fig. 10 the total configurational energy, $U_{\mathrm{T}}^{*}$ and its dipolar con- 
tribution, $U_{\mathrm{D}}^{*}$, as functions of the parallel pressure for the two wall boundary conditions. At $\epsilon_{\mathrm{W}}=1$ the total energy increases monotonously upon compression of the system, indicating that the increase of short-range repulsion dominates the decrease of the dipolar energy (see inset of Fig. 10). In particular there is no sudden decay of $U_{\mathrm{T}}^{*}$ such as the one observed at $L_{\mathrm{z}}^{*}=7.0$ (see Fig. 4) in the vicinity of the isotropic-to-ferroelectric transition. The results obtained at $\epsilon_{\mathrm{W}}=\infty$ reveal a similar trend, with the energies at larger pressures being slightly larger than in the insulating case. A complete list of thermodynamic data at the examplary state $P_{\|}^{*}=2.0$ is given in Table III. Taken altogether, the MC results at $L_{\mathrm{z}}^{*}=2.20$ suggest that the wall boundary conditions do not affect the system's behavior on a qualitative level, whereas the quantitative differences appear to be somewhat larger than those found at $L_{\mathrm{z}}^{*}=7.0$.

\section{LATTICE CALCULATIONS}

We now turn back to the ferroelectric transition occuring in the thicker systems at $L_{\mathrm{z}}^{*}=$ 7.0. The electrostatic arguments presented in Sec. III A [see Eq. (3.2) and below] already provide some explanation of the ordering behavior of the present system with $\epsilon_{\mathrm{W}}=\infty$, in particular its similarity to the case $\epsilon_{\mathrm{W}}=1$. Indeed, according to these arguments there should not be any difference between the two wall boundary conditions because, for both systems, the macroscopic fields in the ordered state are the same.

However, these electrostatic arguments clearly rely on the assumption that macroscopic theory remains to be valid for the nanoscopic fluid slabs considered here. As an attempt to better understand the ordering behavior on a microscopic level we have carried out some additional calculations of the energy of crystalline dipolar slabs with perfect orientational order (and $\epsilon_{\mathrm{W}}=\infty$ ). Making this restriction gives us the possibility to evaluate, based on Eqs. (2.14) and (2.15), the corresponding energies on a quasi-exact level and to compare them with corresponding energies of systems between insulating walls [27].

Specifically, we have considered (infinitely extended) slabs composed of dipolar particles located at the sites of a face-centered cubic (fcc) lattice with lattice vectors $\boldsymbol{r}_{\mathrm{fcc}}=$ $(a / 2)\left(l_{\mathrm{x}}, l_{\mathrm{y}}, l_{\mathrm{z}}\right)$, where $a$ is the lattice constant (fixed such that the reduced density $\rho_{\mathrm{fcc}} \sigma^{3}=$ $\left.4 /(a / \sigma)^{3}=1.0\right)$, and $\left\{l_{\alpha}\right\}(\alpha=\mathrm{x}, \mathrm{y}, \mathrm{z})$ are integers with $l_{\mathrm{x}}+l_{\mathrm{y}}+l_{\mathrm{z}}$ even. An infinitely extended slab is then realized by setting $-\infty<l_{\mathrm{x}}, l_{\mathrm{y}}<\infty$ and $l_{\mathrm{z}}=0, \ldots, n_{\mathrm{z}}-1, n_{\mathrm{z}}$ being 
the number of fcc layers in $z$-direction. Based on this positional structure we have employed the energy relation (2.14) combined with the Ewald expression (2.15) to calculate the total dipolar energy $U_{\mathrm{D}}$ for two dipole configurations characterized by perfect orientational order: (i) all dipoles point along the $x$-axis, that is, $\hat{\mathbf{d}} \| \hat{\mathbf{x}}$ (which is energetically equivalent to $\hat{\mathbf{d}} \| \hat{\mathbf{y}}$ ) and (ii), all dipoles point along the $z$-axis $(\hat{\mathbf{d}} \| \hat{\mathbf{z}})$.

Numerical results for the reduced dipolar energy $U_{\mathrm{D}} \sigma^{3} / \mu^{2} / N$ as function of the number of lattice layers $n_{\mathrm{z}}$ are given in Fig. 11, where part (a) compares the energies related to cases (i) and (ii) in presence of conducting walls $\left(\epsilon_{\mathrm{W}}=\infty\right)$. Regardless of the actual value of $n_{\mathrm{z}}$, the energy related to an ordering parallel to the walls $(\hat{\mathbf{d}} \| \hat{\mathbf{x}})$ is smaller than that related to perpendicular ordering. This is consistent with the macroscopic arguments in Sec. III A and explains why the MC simulations at $L_{\mathrm{z}}^{*}=7$ predict spontaneous polarization parallel to the walls. However, we also observe from Fig. 11 (a) that the actual differences between the two ordering directions are large only for very thin films and decrease with increasing film thickness. Indeed, in the limit $n_{\mathrm{z}} \rightarrow \infty$ both energies converge to the exact value $U_{\mathrm{D}} \sigma^{3} / \mu^{2} / N=-(2 \pi / 3) \rho_{\mathrm{fcc}} P_{1}^{2} \approx-2.094$ (with $\rho_{\mathrm{fcc}}=P_{1}=1$ valid for perfectly ordered bulk dipolar fcc-lattice [44]). This is in marked contrast to the lattice energies of systems with insulating walls $\left(\epsilon_{\mathrm{W}}=1\right)$ [27] for which perpendicular ordering $(\hat{\mathbf{d}} \| \hat{\mathbf{z}})$ turns out to be energetically unfavorable even for $n_{\mathrm{z}} \rightarrow \infty$. We can understand these differences as a consequence of depolarizing fields [see Eq. (3.2)] which arise for perpendicular ordering between insulating walls even in the limit $n_{\mathrm{z}} \rightarrow \infty$ [27].

Finally, we compare in Fig. 11 (b) the energies related to parallel ordering $(\hat{\mathbf{d}} \| \hat{\mathbf{x}})$ for systems between metallic and insulating walls. The latter system is generally characterized by smaller energy values (as compared to the metallic case) which is consistent with our computer simulation results in the ferroelectric phase at $L_{\mathrm{z}}^{*}=7.0$ (see Table II). One also sees that the wall boundary conditions have a large effect only at very small values of $n_{\mathrm{z}}$ where $U_{\mathrm{D}}$ has negative values in the case $\epsilon_{\mathrm{W}}=1$, but not for $\epsilon_{\mathrm{W}}=\infty$. Therefore, ferroelectric (in-plane-) ordering of systems close to two dimensions is even more unlikely in presence of conducting walls, which may explain the absence of ferroelectric ordering in our MC simulations at $L_{\mathrm{z}}^{*}=2.20$ (see Sec. III B). 


\section{SUMMARY AND CONCLUSIONS}

In this paper we have presented an Ewald summation method suitable for computer simulations and lattice calculations of systems of permanent point dipoles confined between conducting walls. The long-range dipolar interactions, including contributions from the "image dipoles" in the metal, are handled by mapping onto a problem with three-dimensional periodicity which can be treated by conventional Ewald summation methods. The final relations between the original and the artificial system are formally the same as those previously found for systems of point charges $[33,34]$. The present method can also be viewed as a generalization of an earlier approach [31] to simulate electrorheological fluids, where all (field-induced) dipoles point perpendicular to the metallic walls, to the case of permanent (and therefore fluctuating) dipoles.

As an illustration of the method we have carried out Monte Carlo computer simulations in order to explore the influence of conducting walls on the structural and phase properties of a dipolar soft sphere fluid confined in a slab. Particular emphasis has been given on the question to which extent the behavior of the present systems differs from the related case of a fluid between non-polarizable walls [27]. Interestingly, the effect of the wall boundaries turns out be very small even for strongly confined fluids and when the wall separations is nanoscopically small. In particular, orientational ordering phenomena such as the isotropicto-ferroelectric transition appearing at larger wall separations (such as, e.g., $L_{\mathrm{z}}^{*}=7.0$ ) and the absence of the latter in systems close to two dimensions $\left(L_{\mathrm{z}}^{*}=2.2\right)$ seem to be unaffected by the nature of the confining walls for the fluids considered here. Differences only arise when one compares numerical values for thermodynamic data such as energies, (normal) pressures, and order parameter, with these differences becoming somewhat more pronounced as the wall separation decreases. We have partially explained this similarity on the basis of lattice calculations involving dipolar crystalline slabs with various types of perfect orientational order. The results (which were based on the Ewald methods) showed that both insulating and conducting walls favor ferroelectric ordering parallel the walls for sufficiently large wall separations, with the energies in the case of conducting walls being slightly larger. Our MC results for the ferroelectric systems at $L_{\mathrm{z}}^{*}=7.0$ are consistent with this picture.

Finally, it seems worth to note that the simulations with conducting walls, which were 
based on the extended three-dimensional Ewald sum proposed in the present work, required significantly less computational time than comparable simulations involving insulating walls (and the slab-adapted version of the Ewald method described in Refs. [22, 27]). Indeed, for the strongly coupled systems discussed in this work the difference (in required CPU time) was about a factor two. From that perspective, and in view of the observed insensitivity of the numerical data against the wall boundary conditions, conducting walls actually appear as a particularly efficient way to simulate confined dipolar systems.

\section{Acknowledgments}

Financial support from the Deutsche Forschungsgemeinschaft via the Sonderforschungsbereich 448 "Mesoskopisch strukturierte Verbundsysteme" and the Emmy-Noether Programme is gratefully acknowledged.

\section{APPENDIX A: RELATION BETWEEN THE ORIGINAL AND THE EX- TENDED SYSTEM}

In this appendix we give a short derivation of Eq. (2.14) which relates the energy of a slab-like system of point dipoles between conducting walls to that of an extended system with three-dimensional periodicity. The basic cell of the extended system contains the $N$ dipoles in the original cell plus the first set of images, e.g., the $N$ images resulting from the presence of the lower wall alone. Positions and orientations of these $N$ image particle are then specified by the relations [see Eq. (2.5) with $n_{\mathrm{z}}=0$ and Eq. (2.4)]

$$
\begin{aligned}
\mathbf{r}_{i+N} & =\mathbf{r}_{i}-2 z_{i} \hat{\mathbf{z}}, \quad i=1, \ldots, N \\
\boldsymbol{\mu}_{i+N} & =\boldsymbol{\mu}_{i}^{\prime}=\left(-\mu_{i, \mathrm{x}},-\mu_{i, \mathrm{y}}, \mu_{i, \mathrm{z}}\right), \quad i=1, \ldots, N
\end{aligned}
$$

Repeating the extended basic cell periodically in all three spatial directions, the total energy is given as

$$
U_{\mathrm{D}}^{\mathrm{ex}, 3 \mathrm{~d}}=\frac{1}{2} \sum_{i=1}^{2 N} \sum_{j=1}^{2 N} \sum_{\overline{\boldsymbol{n}}}^{\prime}\left[\frac{\boldsymbol{\mu}_{i} \cdot \boldsymbol{\mu}_{j}}{\left|\boldsymbol{r}_{i j}+\overline{\boldsymbol{n}}\right|^{3}}-3 \frac{\left(\boldsymbol{\mu}_{i} \cdot\left(\boldsymbol{r}_{i j}+\overline{\boldsymbol{n}}\right)\right)\left(\boldsymbol{\mu}_{j} \cdot\left(\boldsymbol{r}_{i j}+\overline{\boldsymbol{n}}\right)\right)}{\left|\boldsymbol{r}_{i j}+\overline{\boldsymbol{n}}\right|^{5}}\right],
$$

where the lattice vectors $\overline{\boldsymbol{n}}$ are specified in Eq. (2.12), and the prime at the sum indicates that the term related to $i=j$ is omitted for $\overline{\boldsymbol{n}}=0$. We now separate the double sum 
where $\boldsymbol{r}_{i+N, j+N}=\boldsymbol{r}_{i+N}-\boldsymbol{r}_{j+N}$,

$$
\begin{aligned}
& \left(\boldsymbol{\mu}_{i+N} \cdot\left(\boldsymbol{r}_{i+N, j+N}+\overline{\boldsymbol{n}}\right)\right)\left(\boldsymbol{\mu}_{j+N} \cdot\left(\boldsymbol{r}_{i+N, j+N}+\overline{\boldsymbol{n}}\right)\right) \\
= & \left(\boldsymbol{\mu}_{i} \cdot\left(\boldsymbol{r}_{i j}+\overline{\boldsymbol{n}}-4 n_{\mathrm{z}} L_{\mathrm{z}} \hat{\mathbf{z}}\right)\right)\left(\boldsymbol{\mu}_{j} \cdot\left(\boldsymbol{r}_{i j}+\overline{\boldsymbol{n}}-4 n_{\mathrm{z}} L_{\mathrm{z}} \hat{\mathbf{z}}\right)\right),
\end{aligned}
$$

and the fact that we sum in Eq. (A2) over an infinite set of lattive vectors $\{\overline{\boldsymbol{n}}\}$ such that the terms $-4 L_{\mathrm{z}} n_{\mathrm{z}} \hat{\mathbf{z}}$ appearing on the right side of Eqs. (A3) and (A4) are irrelevant.

Similarly, the lattice sums over terms (iii) and (iv) in the above decomposition also give equivalent results due to the relations

$$
\begin{aligned}
\boldsymbol{\mu}_{i} \cdot \boldsymbol{\mu}_{j+N} & =\boldsymbol{\mu}_{i+N} \cdot \boldsymbol{\mu}_{j} \\
\left|\boldsymbol{r}_{i, j+N}+\overline{\boldsymbol{n}}\right| & =\left|\boldsymbol{r}_{i+N, j}+\overline{\boldsymbol{n}}-4 n_{\mathrm{z}} L_{\mathrm{z}} \hat{\mathbf{z}}\right|
\end{aligned}
$$

and

$$
\begin{aligned}
& \left(\boldsymbol{\mu}_{i} \cdot\left(\boldsymbol{r}_{i, j+N}+\overline{\boldsymbol{n}}\right)\right)\left(\boldsymbol{\mu}_{j+N} \cdot\left(\boldsymbol{r}_{i, j+N}+\overline{\boldsymbol{n}}\right)\right) \\
= & \left(\boldsymbol{\mu}_{i+N} \cdot\left(\boldsymbol{r}_{i+N, j}+\overline{\boldsymbol{n}}-4 n_{\mathrm{z}} \hat{\mathbf{z}}\right)\right)\left(\boldsymbol{\mu}_{j} \cdot\left(\boldsymbol{r}_{i+N, j}+\overline{\boldsymbol{n}}-4 n_{\mathrm{z}} \hat{\mathbf{z}}\right)\right) .
\end{aligned}
$$

Due to these symmetries we can rewrite Eq. (A2) as

$$
\begin{aligned}
U_{\mathrm{Dip}}^{\mathrm{ex}, 3 \mathrm{~d}}= & \sum_{i=1}^{N} \sum_{j=1}^{N}\left\{\sum_{\overline{\boldsymbol{n}}}^{\prime}\left[\frac{\boldsymbol{\mu}_{i} \cdot \boldsymbol{\mu}_{j}}{\left|\boldsymbol{r}_{i j}+\overline{\boldsymbol{n}}\right|^{3}}-3 \frac{\left(\boldsymbol{\mu}_{i} \cdot\left(\boldsymbol{r}_{i j}+\overline{\boldsymbol{n}}\right)\right)\left(\boldsymbol{\mu}_{j} \cdot\left(\boldsymbol{r}_{i j}+\overline{\boldsymbol{n}}\right)\right)}{\left|\boldsymbol{r}_{i j}+\overline{\boldsymbol{n}}\right|^{5}}\right]\right. \\
& \left.+\sum_{\overline{\boldsymbol{n}}}\left[\frac{\boldsymbol{\mu}_{i} \cdot \boldsymbol{\mu}_{j+N}}{\left|\boldsymbol{r}_{i, j+N}+\overline{\boldsymbol{n}}\right|^{3}}-3 \frac{\left(\boldsymbol{\mu}_{i} \cdot\left(\boldsymbol{r}_{i, j+N}+\overline{\boldsymbol{n}}\right)\right)\left(\boldsymbol{\mu}_{j+N} \cdot\left(\boldsymbol{r}_{i, j+N}+\overline{\boldsymbol{n}}\right)\right)}{\left|\boldsymbol{r}_{i, j+N}+\overline{\boldsymbol{n}}\right|^{5}}\right]\right\} .
\end{aligned}
$$

Noting that $\boldsymbol{\mu}_{j+N}=\boldsymbol{\mu}_{j}^{\prime}$ and $\boldsymbol{r}_{i, j+N}=\boldsymbol{r}_{i}-\boldsymbol{r}_{j+N}=\boldsymbol{r}_{i}-\boldsymbol{r}_{j}+2 z_{j} \hat{\mathbf{z}}$ [see Eqs. (2.4) and (A1)], one realizes that the energy of the three-dimensional extended system given in Eq. (A7) is indeed exactly twice the energy $U_{\mathrm{D}}$ defined in Eq. (2.11). 


\section{APPENDIX B: PRESSURE TENSOR}

In the following we present explicit expressions for the diagonal components $P_{\gamma \gamma}$ (with $\gamma=\mathrm{x}, \mathrm{y}$, or $\mathrm{z}$ ) of the pressure tensor, $\mathbf{P}$, for a dipolar fluid confined between metallic walls. Considering first a canonical ensemble (i.e., constant $N, T, A$, and $L_{\mathrm{z}}$ ), the virial formula for $P_{\gamma \gamma}[45]$ gives

$$
P_{\gamma \gamma}=\beta^{-1} \frac{N}{A L_{\mathrm{z}}}-\frac{1}{A_{\gamma}}\left\langle\frac{\partial U_{\mathrm{T}}}{\partial L_{\gamma}}\right\rangle,
$$

where $A_{\gamma}=V_{\text {cell }} / L_{\gamma}$, and $U_{\mathrm{T}}$ is the total energy including both fluid-fluid and fluid-wall interactions [see Eq. (2.18)]. We note that the $L_{\gamma}$-derivatives in Eq. (B1) have to be performed at fixed scaled coordinates $u_{i}=r_{i, \gamma} / L_{\gamma}$ with $r_{i, \gamma}$ being the $\gamma$-component of the position vectors $\boldsymbol{r}_{i}$ (see Ref. [45] for an examplary derivation of the normal component).

Because of the simple structure of the short-range and fluid-wall potential [see Eqs. (2.2) and (2.3), respectively] the corresponding pressure contributions can be easily derived (note that the fluid-wall potential only contributes to $P_{\mathrm{zz}}$ ). For the evaluation of the dipolar contribution,

$$
P_{\mathrm{D}, \gamma \gamma}=-\frac{1}{A_{\gamma}}\left\langle\frac{\partial U_{\mathrm{D}}}{\partial L_{\gamma}}\right\rangle
$$

we make use of the energy mapping relation (2.14) which implies

$$
P_{\mathrm{D}, \gamma \gamma}=-\frac{1}{A_{\gamma}} \frac{1}{2}\left\langle\frac{\partial U_{\mathrm{D}}^{3 \mathrm{~d}, \mathrm{ex}}}{\partial L_{\gamma}}\right\rangle=\frac{1}{2}\left(P_{\gamma \gamma}^{\mathrm{real}}+\Delta P_{\mathrm{D}, \gamma \gamma}\right) .
$$

Thus, the dipolar contribution to the pressure tensor of the present system can be calculated from the corresponding formulae for three-dimensional (3d) dipolar systems with extended system size (see Appendix A). A detailed derivation of the real-space contribution, $P_{\gamma \gamma}^{\text {real }}$, and the reciprocal contribution, $\Delta P_{\mathrm{D}, \gamma \gamma}$, for the 3d Ewald sum [see Eq. (2.15)] can be found, e.g., in Refs. [46]. One obtains

$$
\begin{aligned}
P_{\gamma \gamma}^{\text {real }}= & \frac{1}{2 V_{\text {cell }}^{\mathrm{ex}}}\left\langle\sum _ { i = 1 } ^ { 2 N } \sum _ { j \neq i } \left\{C ( r _ { i j } , \alpha ) \left[\left(\boldsymbol{\mu}_{i} \cdot \boldsymbol{\mu}_{j}\right) r_{i j, \gamma}^{2}\right.\right.\right. \\
& \left.+\mu_{i, \gamma} r_{i j, \gamma}\left(\boldsymbol{\mu}_{j} \cdot \boldsymbol{r}_{i j}\right)+\left(\boldsymbol{\mu}_{i} \cdot \boldsymbol{r}_{i j}\right) \mu_{j, \gamma} r_{i j, \gamma}\right] \\
& \left.\left.+\left(\boldsymbol{\mu}_{i} \cdot \boldsymbol{r}_{i j}\right)\left(\boldsymbol{\mu}_{j} \cdot \boldsymbol{r}_{i j}\right) r_{i j, \gamma} \tilde{C}\left(r_{i j}, \alpha\right)\right\}\right\rangle
\end{aligned}
$$

where $\tilde{r}_{i j, \gamma}$ is the $\gamma$-component of vector $\boldsymbol{r}_{i j}=\boldsymbol{r}_{i}-\boldsymbol{r}_{j}$, and the function $\tilde{C}\left(r_{i j}, \alpha\right)=$ $r_{i j}^{-1} \partial C / \partial r_{i j}$ [cf. Eq. (2.16)]. Furthermore, the reciprocal part of the dipolar pressure tensor 


$$
\begin{aligned}
\Delta P_{\gamma \gamma}^{\mathrm{D}}= & \frac{1}{\left(V_{\mathrm{cell}}^{\mathrm{ex}}\right)^{2}}\left\langle\sum_{\boldsymbol{k} \neq \mathbf{0}} \frac{4 \pi}{k^{2}} \exp \left(-\frac{k^{2}}{4 \alpha^{2}}\right)\right. \\
& \times\left\{\left(1-\frac{2 k_{\gamma}^{2}}{k^{2}}-\frac{k_{\gamma}^{2}}{2 \alpha^{2}}\right) \widetilde{M}(\boldsymbol{k}) \widetilde{M}^{*}(\boldsymbol{k})\right. \\
& \left.\left.+\widetilde{Q}(\boldsymbol{k}) \widetilde{M}^{*}(\boldsymbol{k})+\widetilde{Q}^{*}(\boldsymbol{k}) \widetilde{M}(\boldsymbol{k})\right\}\right\rangle
\end{aligned}
$$

where the quantity $\widetilde{M}(\boldsymbol{k})$ is defined in Eq. (2.17), and

$$
\widetilde{Q}(\boldsymbol{k})=\sum_{i=1}^{2 N} \mu_{i, \gamma} k_{\gamma} \exp \left(i \boldsymbol{k} \cdot \boldsymbol{r}_{i}\right)
$$

A generalization of the above virial formulae for the (dipolar) pressure tensor to systems simulated in the $\left(N, L_{\mathrm{z}}, P_{\|}, L_{\mathrm{z}}\right)$ ensemble was presented by us in Ref. [27]. The normal pressure $P_{\mathrm{zz}}$ then follows from the canonical formulae via a simple replacement of the area $A$ by its average, $\langle A\rangle$.

The parallel contributions, $\tilde{P}_{\mathrm{xx}}$ and $\tilde{P}_{\mathrm{yy}}$, do not have any rigourous statistical expression in the $\left(N, L_{\mathrm{z}}, P_{\|}, L_{\mathrm{z}}\right)$ ensemble, but their evaluation might still be useful as an internal check of the simulations. Defining these components through the relations [27]

$$
\tilde{P}_{\gamma \gamma}=\beta^{-1} \frac{N}{\langle A\rangle L_{\mathrm{z}}}-\frac{1}{\langle A\rangle L_{\mathrm{z}}}\left\langle L_{\gamma} \frac{\partial U_{\mathrm{T}}}{\partial L_{\gamma}},\right\rangle, \quad \gamma=\mathrm{x}, \mathrm{y}
$$

and performing the derivatives one arrives again at the canonical formula given above with the replacement $A \rightarrow\langle A\rangle$.

[1] M. Hara, Polyelectrolytes: Science and Technology, Dekker, New York (1993).

[2] S. H. L. Klapp. Dipolar fluids under external perturbations, J. Phys.: Condens. Matter, 17, R525 (2005).

[3] E. Blums, A. Cebers, M. M. Maiorov. Magnetic Fluids, Walter de Gruyter, New York (1997).

[4] S. Odenbach. Ferrofluids, Magnetically Controllable Fluids and their Applications, Lecture Notes in Physics, vol. 594, Springer, Berlin (2002).

[5] R. E. Rosensweig. Ferrohydrodynamics, Cambridge University Press, Cambridge (1985).

[6] T. C. Halsey, in Electrorheological Fluids, edited by R. Tao, World Scientific, Singapore (1992). 
[7] C. Stubenrauch, R. von Klitzing. Disjoining pressure in thin liquid foam and emulsion films new concepts and perspectives. J. Phys.: Condens. Matter, 15, R1197 (2003).

[8] M. Seul, R. Wolfe. Competing interactions and domain-shape instabilities in a monomolecular film at an air-water interface. Phys. Rev. Lett., 64, 1903 (1990).

[9] G. J. Zarragoicoechea. A model phospholipid monolayer: a computer simulation study. Mol. Phys., 96, 1109 (1999).

[10] J. J. Weis. Simulation of quasi-two-dimensional dipolar systems. J. Phys.: Condens. Matter, 15, S1471 (2003).

[11] S.W. de Leeuw, J.W. Perram, E.R. Smith. Simulation of electrostatic systems in periodic boundary conditions. 1. Lattice sums and dielectric constants. Proc. R. Soc. London, Ser. A, 373, 27 (1980).

[12] S.W. de Leeuw, J.W. Perram, E.R. Smith. Simulation of electrostatic systems in periodic boundary conditions. 3. Further Theory and Applications. Proc. R. Soc. London, Ser. A, 388, 177 (1983).

[13] M.P. Allen, D.J. Tildesley. Computer Simulation of Liquids, Chap. 5.5., Academic, London, (1987).

[14] D. Frenkel, B. Smit. Understanding Molecular Simulation, Academic Press, San Diego (2002).

[15] D. E. Parry. Electrostatic potential in surface region of an ionic crystal. Surf. Sci., 49, 433 (1975)

[16] D. E. Parry. Correction. Surf. Sci., 54, 195 (1976).

[17] S.W. de Leeuw, J.W. Perram. Electrostatic lattice sums for semi-infinite lattices. Mol. Phys., 37, 1313 (1979).

[18] D. M. Heyes, F. van Swol. The electrostatic potential and field in the surface region of lamina and semi-infinite point charge lattices. J. Chem. Phys., 75, 5051 (1981).

[19] Y. J. Rhee, J. W. Halley, J. Hautman, A. Rahman. Ewald methods in molecular dynamics for systems of finite extent in one of three dimensions. Phys. Rev. B, 40, 36 (1989).

[20] J. Hautman, M. L. Klein. An Ewald summation method for planar surfaces and interfaces. Mol. Phys., 92, 379 (1991).

[21] A. Grzybowski, E. Gwozdz, A. Brodka. Ewald summation of electrostatic interactions in molecular dynamics of a three-dimensional system with periodicity in two directions. Phys. Rev. B, 61, 6706 (2000). 
[22] I.-C. Yeh, M. L. Berkowitz. Ewald summation for systems with slab geometry. J. Chem. Phys., 111, 3155 (1999).

[23] J. C. Shelley, G. N. Patey. Boundary condition effects in simulations of water confined between planar walls. Mol. Phys., 88, 385 (1996).

[24] E. Spohr. Effect of electrostatic boundary conditions and system size on the interfacial properties of water and aqueous solutions. J. Chem. Phys., 107, 6342 (1997).

[25] P. S. Crozier, R. L. Rowley, E. Spohr, D. Henderson. Comparison of charged sheets and corrected 3D Ewald calculations of long-range forces in slab geometry electrolyte systems with solvent molecules. J. Chem. Phys., 112, 9253 (2000).

[26] A. Arnold, J. de Joannis, C. Holm. Electrostatics in periodic slab geometries. I, J. Chem. Phys., 117, 2496 (2002).

[27] S. Klapp, M. Schoen. Spontaneous orientational order in confined dipolar fluid films. J. Chem. Phys., 117, 8050 (2002).

[28] S. Klapp, M. Schoen. Ferroelectric states of a dipolar fluid confined to a slit-pore. J. Mol. Liq., 109, 55 (2004).

[29] V. Ballenegger, J.-P. Hansen. Dielectric permittivity profiles of confined polar fluids. J. Chem. Phys., 122, 114711 (2005).

[30] D. R. Berard, M. Kinoshita, X. Ye, G. N. Patey. Structure and properties of the metalliquid interface. J. Chem. Phys., 101, 6271 (1994).

[31] G. L. Gulley. R. Tao, Structures of an electrorheological fluid, Phys. Rev E, 56, 4328 (1997).

[32] J. D. Jackson, Classical Electrodynamics, 3rd ed., John Wiley, New York (1999).

[33] J. Hautman, J. W. Halley, Y.-J. Rhee. Molecular dynamics simulation of water beween two ideal classical metal walls. J. Chem Phys., 91, 467 (1989).

[34] J. W. Perram, M. A. Ratner. Simulations at conducting interfaces: Boundary conditions for electrodes and electrolytes. J. Chem Phys., 104, 5174 (1996).

[35] M. Schoen. The Joule-Thomson effect in confined fluids. Physica A, 270, 353 (1999).

[36] D. Wei, G.N. Patey. Orientational order in simple dipolar liquids: Computer simulation of a ferroelectric nematic phase. Phys. Rev. Lett., 68, 2043 (1992).

[37] D. Wei, G.N. Patey. Ferroelectric liquid-crystal and solid phases formed by strongly interacting dipolar soft spheres. Phys. Rev. A, 46, 7783 (1992).

[38] J. J. Weis, D. Levesque, G. J. Zarragoicoechea. Orientational order in simple dipolar liquid- 
crystal models. Phys. Rev. Lett., 69, 913 (1992).

[39] J. J. Weis, D. Levesque. Ferroelectric phases of dipolar hard spheres. Phys. Rev. E, 48, 3728 (1993).

[40] R. Eppenga, D. Frenkel. Monte Carlo study of the isotropic and nematic phases of infinitely thin hard platelets. Mol. Phys., 52, 1303 (1984).

[41] C. F. Böttcher. Theory of Electric Polarization, 2nd ed., Elsevier, New York (1973).

[42] W. F. Brown, Jr., in Handbuch der Physik, Vol. XVII., edited by S. Flügge, Springer, Berlin (1956).

[43] P. I. C. Teixeira, J. M. Tavares, M. M. Telo da Gama. The effect of dipolar forces on the structure and thermodynamics of classical fluids. J. Phys: Condens. Mat., 12, R411 (2000).

[44] J. M. Luttinger, L. Tisza. Theory of Dipole Interaction in Crystals. Phys. Rev., 70, 954 (1946).

[45] M. Schoen, in Computational Methods in Surface and Colloid Science, edited by M. Borowko, Marcel Dekker Inc. (2000).

[46] P. R. ten Wolde, D. W. Oxtoby, D. Frenkel. Chain formation in homogeneous gasliquid nucleation of polar fluids. J. Chem. Phys., 111, 4762 (1999). 


\section{FIGURES}

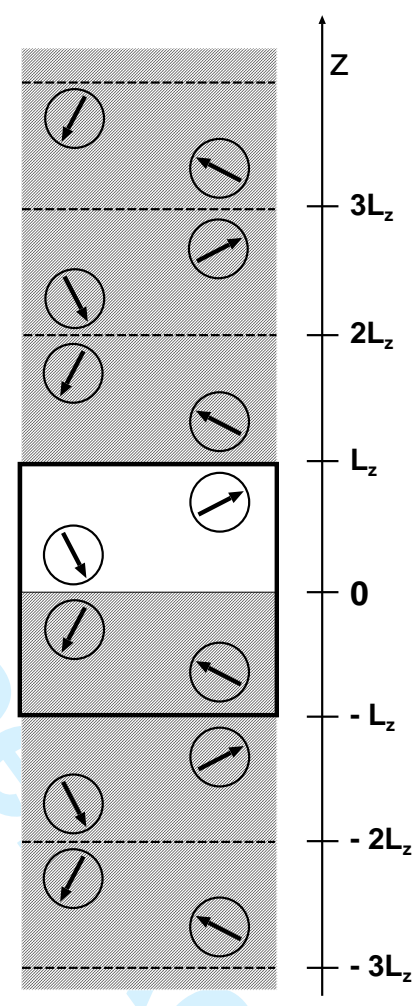

FIG. 1: Sketch of the effect of conducting walls on two dipolar particles located in the white box. The combination of two conducting walls yields an infinite number of images per particle, where one group of images has the same orientations as the original dipole, whereas the other group is characterized by reflected orientations. The structure in $z$-direction can be considered as an infinite periodic replication of the extended cell (original dipoles plus one set of images) marked by the thick frame. 


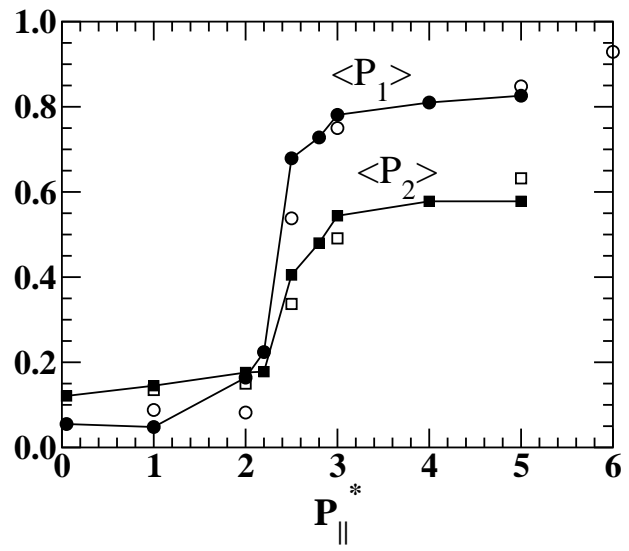

FIG. 2: Order parameters $\left\langle P_{1}\right\rangle$ and $\left\langle P_{2}\right\rangle$ versus parallel pressure $P_{\|}^{*}$ at wall separation $L_{\mathrm{z}}^{*}=7.0$ $\left(T^{*}=1.35\right.$ and $\left.m^{*}=3.0\right)$. The solid and open symbols indicate MC results for conducting and insulating walls [27], respectively.

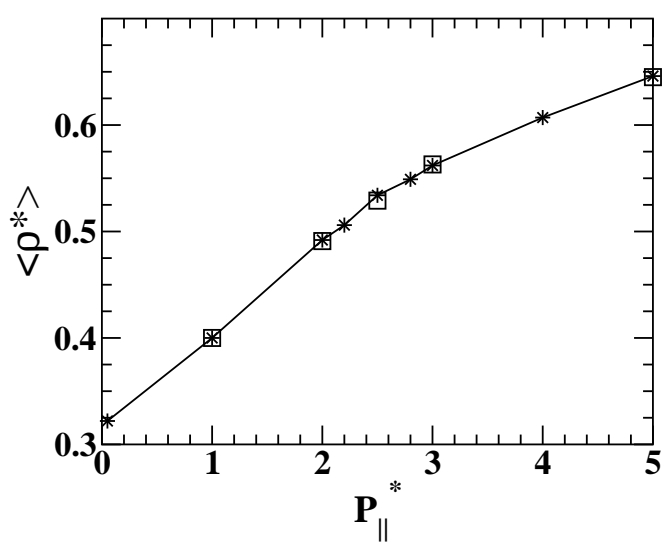

FIG. 3: Density versus parallel pressure $P_{\|}^{*}$ at $L_{\mathrm{z}}^{*}=7.0$. The solid and open symbols indicate results for conducting and insulating walls, respectively. 


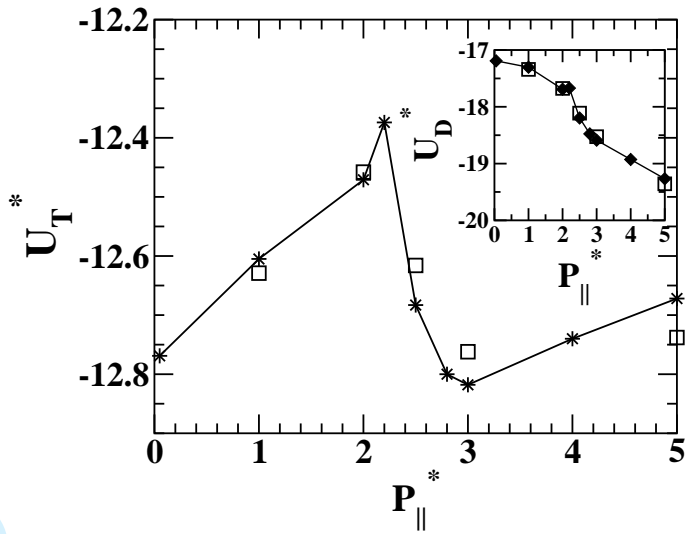

FIG. 4: Energies versus parallel pressure $P_{\|}^{*}$ at $L_{\mathrm{z}}^{*}=7.0$. The solid and open symbols indicate results for conducting and insulating walls [27], respectively.

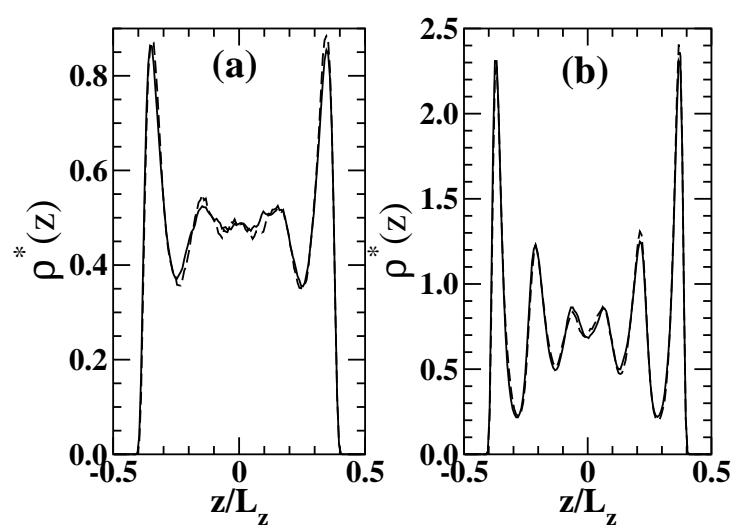

FIG. 5: Local number density at (a) $P_{\|}^{*}=1$ (isotropic phase) and (b) $P_{\|}^{*}=5$ (ferroelectric phase). Results for conducting and insulating walls are indicated by solid and dashed lines, respectively. 

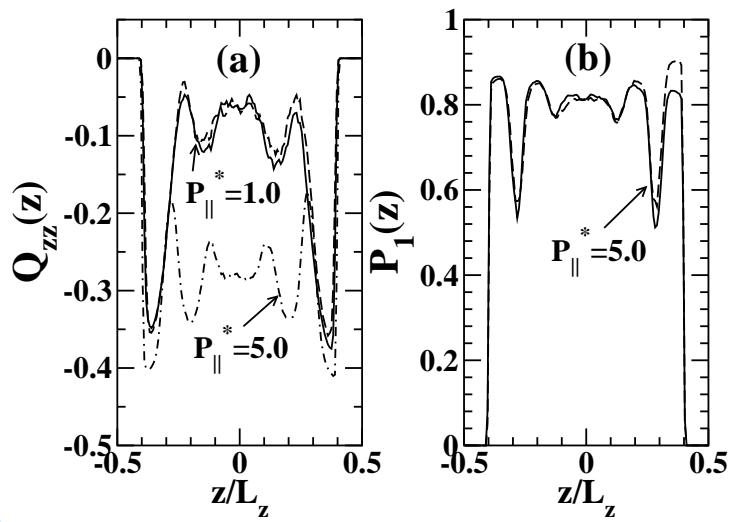

FIG. 6: (a) The local order parameter $Q_{\mathrm{zz}}(z)$ at $P_{\|}^{*}=1.0$ and $P_{\|}^{*}=5.0$. (b) The local polarization at $P_{\|}^{*}=5.0$. Results for conducting and insulating walls are indicated by solid and dashed lines, respectively.
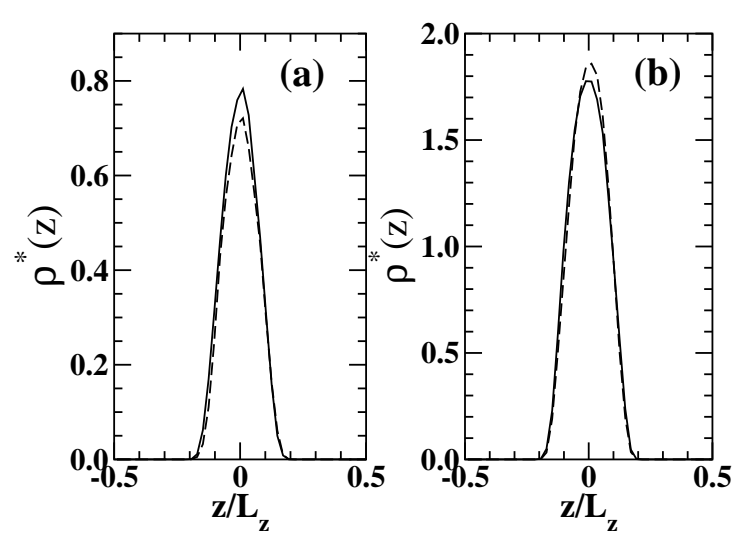

FIG. 7: Local densities at $L_{\mathrm{z}}^{*}=2.20$ and two different parallel pressures $P_{\|}^{*}=0.005$ (a) and $P_{\|}^{*}=2.0$ (b). The solid and dashed lines indicate results for conducting and insulating walls, respectively. 


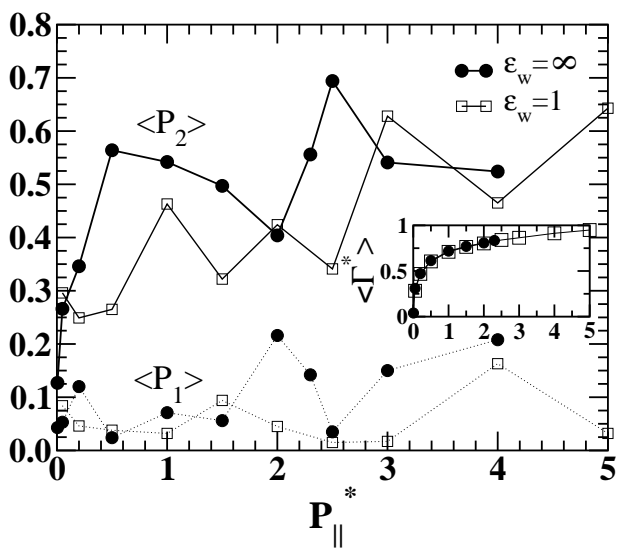

FIG. 8: Order parameters versus parallel pressure $P_{\|}^{*}$ at $L_{\mathrm{z}}^{*}=2.20$. The solid and open symbols indicate results for conducting and insulating walls, respectively.

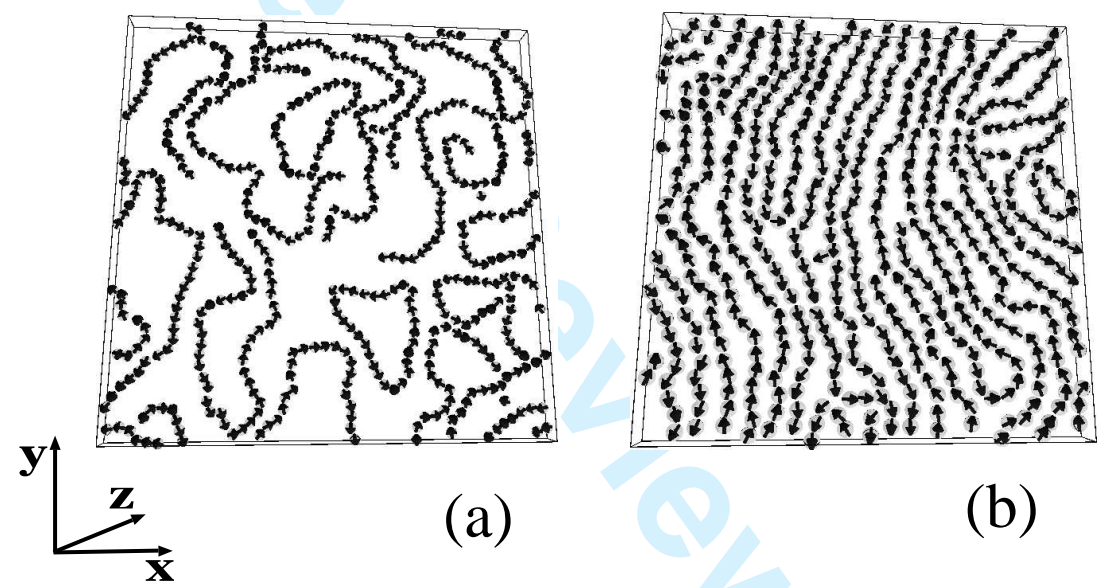

FIG. 9: Snapshots of the system at $L_{\mathrm{z}}^{*}=2.20$ (quasi-monolayer) and $\epsilon_{\mathrm{W}}=\infty$ for two different parallel pressures $P_{\|}^{*}=0.05$ (a) and $P_{\|}^{*}=1.0(\mathrm{~b})$. 


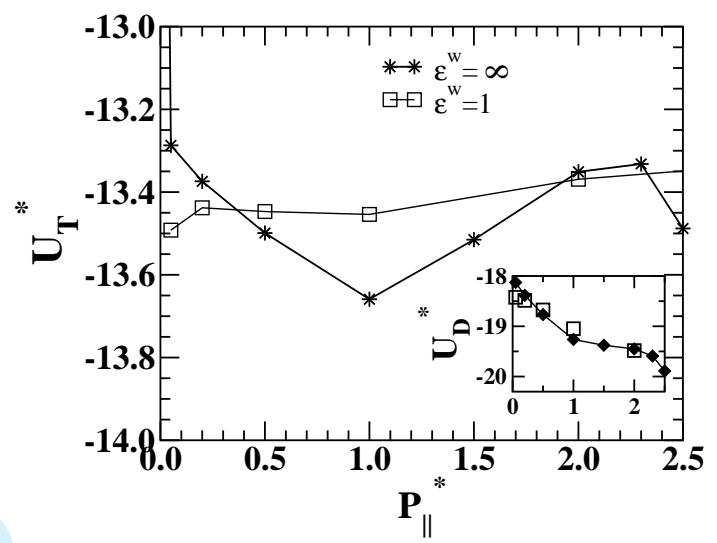

FIG. 10: Energies versus parallel pressure $P_{\|}^{*}$ at $L_{\mathrm{z}}^{*}=2.20$. The solid and open symbols indicate results for conducting and insulating walls, respectively.

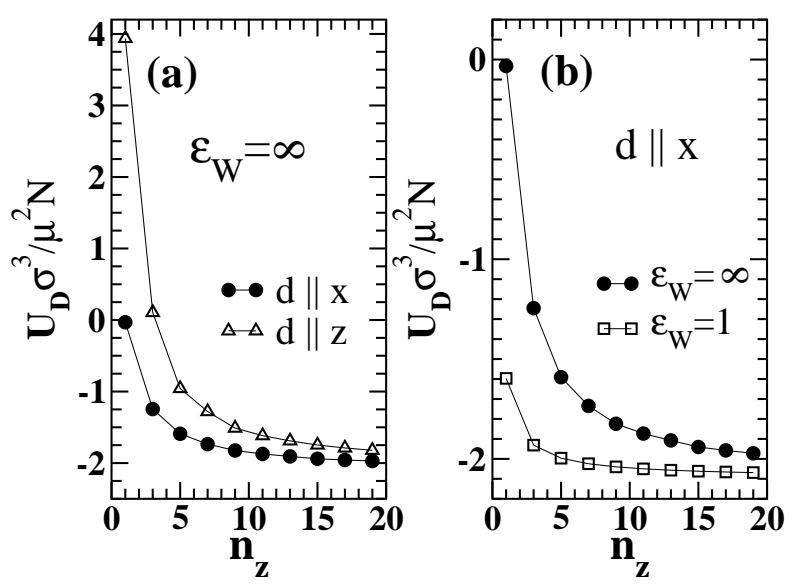

FIG. 11: Dimensionless energy per particle for dipolar crystalline (fcc) slabs with perfect orientational order. Part (a) refers to systems between metallic walls and $\hat{\mathbf{d}} \| \hat{\mathbf{x}}$ or $\hat{\mathbf{d}} \| \hat{\mathbf{z}}$. Part (b) compares for the case $\hat{\mathbf{d}} \| \hat{\mathbf{x}}$ the energies of systems between metallic and insulating walls [27]. 


\section{TABLES}

TABLE I: Simulation results for the average density, the order parameters, the short-ranged (SR), fluid-wall (FW), dipolar (D), and total (T) reduced potential energy $\left[U^{*}=U /\left(\epsilon_{0} N\right)\right]$, and the components $P_{\mathrm{T}, \gamma \gamma}^{*}=P_{\mathrm{T}, \gamma \gamma} \sigma^{3} / \epsilon_{0}$ (with $\gamma=\mathrm{x}, \mathrm{y}, \mathrm{z}$ ) of the reduced (total) pressure tensor for DSS fluids between insulating $\left(\epsilon_{\mathrm{W}}=1\right)$ and conducting walls $\left(\epsilon_{\mathrm{W}}=\infty\right)$ at $T^{*}=1.35, m^{*}=3.0$, $L_{\mathrm{z}}^{*}=7.0$, and $P_{\|}^{*}=1.0$ (isotropic phase).

\begin{tabular}{l|r|r|r|r|r|r|r|r|r|r}
\hline \hline & $\left\langle\rho^{*}\right\rangle$ & $\left\langle P_{1}\right\rangle$ & $\left\langle P_{2}\right\rangle$ & $U_{\mathrm{SR}}^{*}$ & $U_{\mathrm{FW}}^{*}$ & $U_{\mathrm{D}}^{*}$ & $U_{\mathrm{T}}^{*}$ & $\tilde{P}_{\mathrm{T}, \mathrm{xx}}^{*}$ & $\tilde{P}_{\mathrm{T}, \mathrm{yy}}^{*}$ & $P_{\mathrm{T}, \mathrm{zz}}^{*}$ \\
\hline$\epsilon_{\mathrm{W}}=\infty$ & 0.40 & 0.05 & 0.15 & 4.60 & 0.099 & -17.30 & -12.61 & 1.00 & 1.01 & 1.32 \\
$\epsilon_{\mathrm{W}}=1$ & 0.40 & 0.09 & 0.14 & 4.62 & 0.094 & -17.34 & -12.63 & 1.00 & 1.01 & 1.31 \\
\hline \hline
\end{tabular}

TABLE II: Thermodynamic data (for explanation, see Table I) at $T^{*}=1.35, m^{*}=3.0, L_{\mathrm{z}}^{*}=7.0$, and $P_{\|}^{*}=5.0$ (ferroelectric phase).

\begin{tabular}{l|r|r|r|r|r|r|r|r|r|r} 
& $\left\langle\rho^{*}\right\rangle$ & $\left\langle P_{1}\right\rangle$ & $\left\langle P_{2}\right\rangle$ & $U_{\mathrm{SR}}^{*}$ & $U_{\mathrm{FW}}^{*}$ & $U_{\mathrm{D}}^{*}$ & $U_{\mathrm{T}}^{*}$ & $\tilde{P}_{\mathrm{T}, \mathrm{xx}}^{*}$ & $\tilde{P}_{\mathrm{T}, \mathrm{yy}}^{*}$ & $P_{\mathrm{T}, \mathrm{zz}}^{*}$ \\
\hline \hline$\epsilon_{\mathrm{W}}=\infty$ & 0.65 & 0.83 & 0.58 & 6.34 & 0.26 & -19.26 & -12.67 & 4.98 & 5.02 & 5.94 \\
$\epsilon_{\mathrm{W}}=1$ & 0.65 & 0.85 & 0.63 & 6.37 & 0.25 & -19.36 & -12.74 & 5.01 & 4.99 & 5.93 \\
\hline \hline
\end{tabular}

TABLE III: Thermodynamic data (for explanation, see Table I) at $T^{*}=1.35, m^{*}=3.0, L_{\mathrm{z}}^{*}=2.20$, and $P_{\|}^{*}=2.0$.

\begin{tabular}{c|c|r|r|r|r|r|r|r|r|r}
\hline \hline & $N \sigma^{2} /\langle A\rangle$ & $\left\langle P_{1}\right\rangle$ & $\left\langle P_{2}\right\rangle$ & $U_{\mathrm{SR}}^{*}$ & $U_{\mathrm{FW}}^{*}$ & $U_{\mathrm{D}}^{*}$ & $U_{\mathrm{T}}^{*}$ & $\tilde{P}_{\mathrm{T}, \mathrm{xx}}^{*}$ & $\tilde{P}_{\mathrm{T}, \mathrm{yy}}^{*}$ & $P_{\mathrm{T}, \mathrm{zz}}^{*}$ \\
\hline$\epsilon_{\mathrm{W}}=\infty$ & 0.810 & 0.21 & 0.41 & 5.50 & 0.60 & -19.45 & -13.35 & 2.01 & 1.99 & 2.32 \\
$\epsilon_{\mathrm{W}}=1$ & 0.802 & 0.04 & 0.42 & 5.53 & 0.57 & -19.48 & -13.70 & 1.97 & 2.02 & 2.29 \\
\hline \hline
\end{tabular}




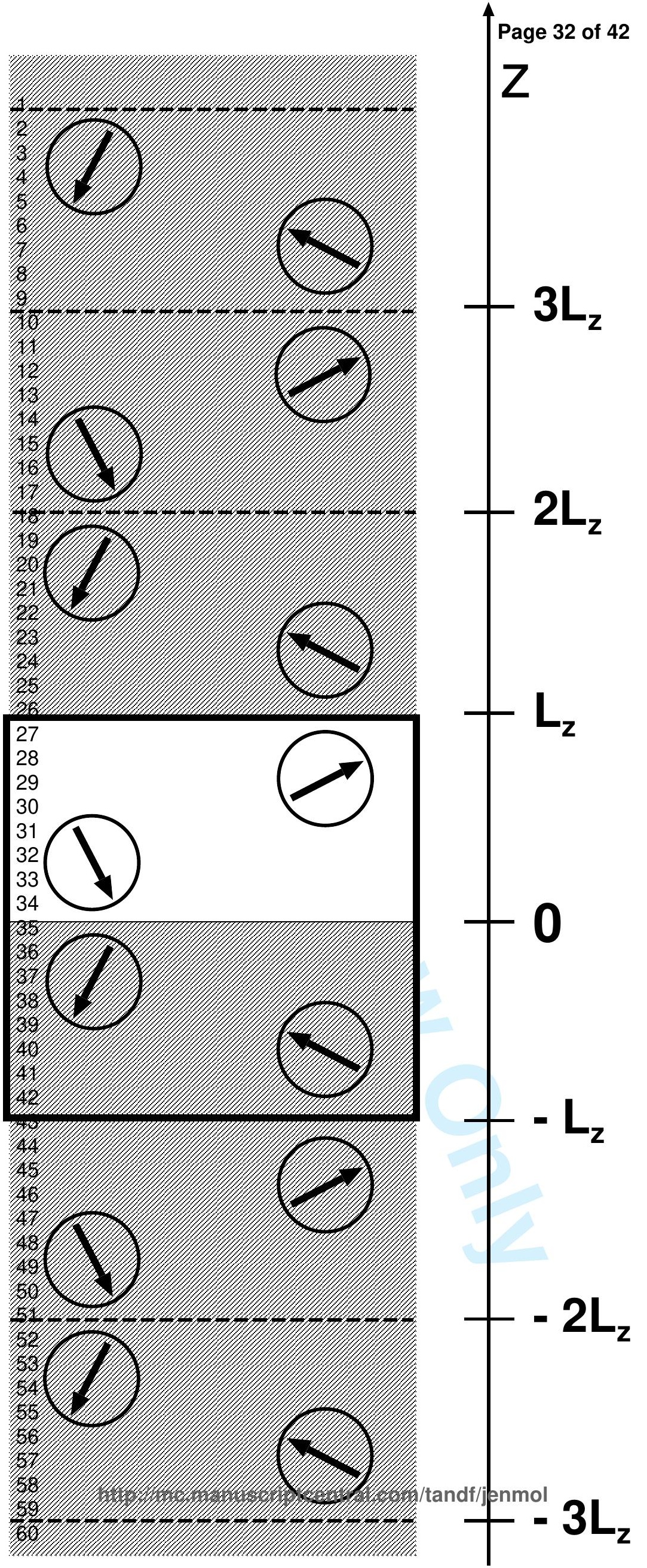




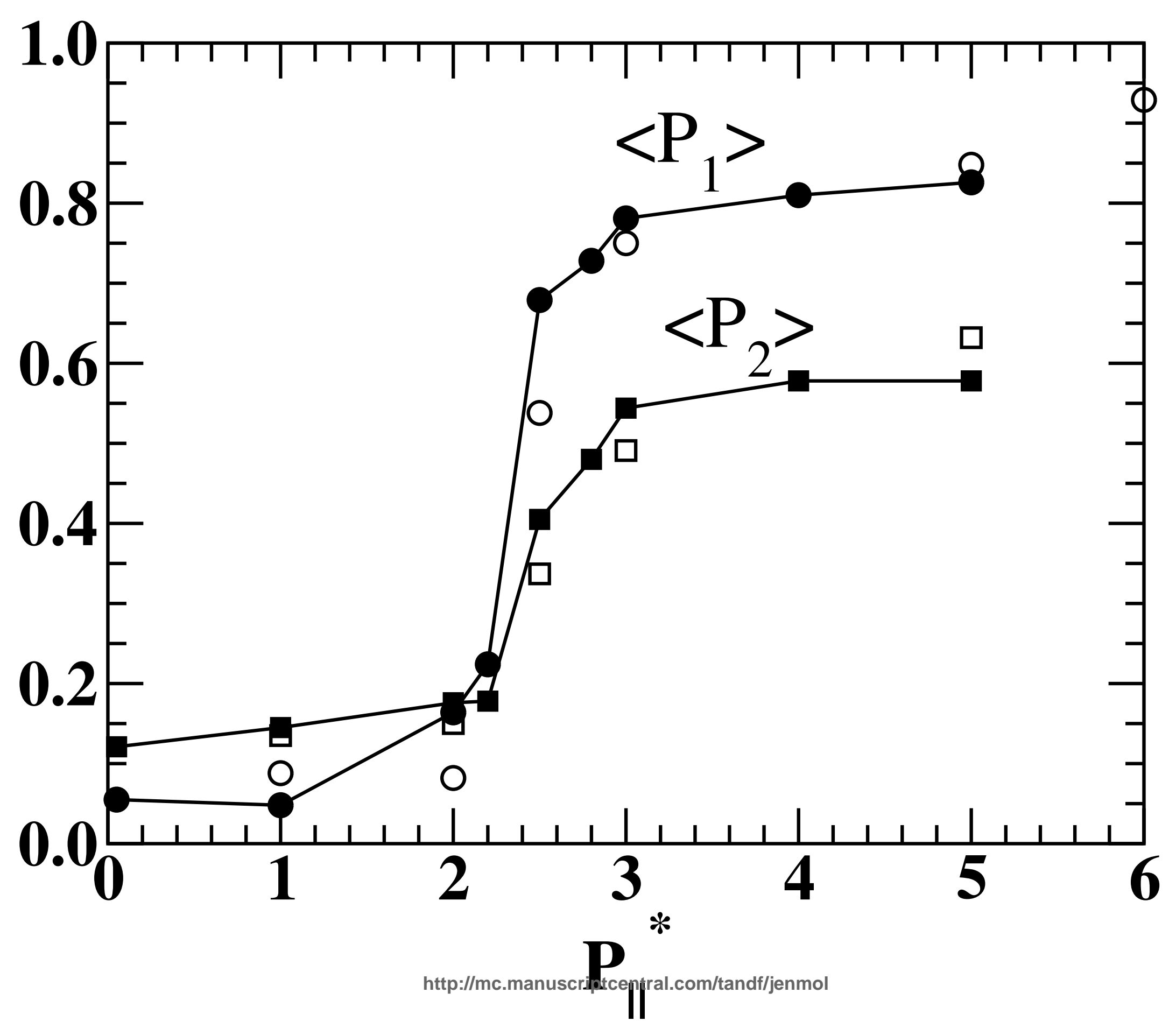




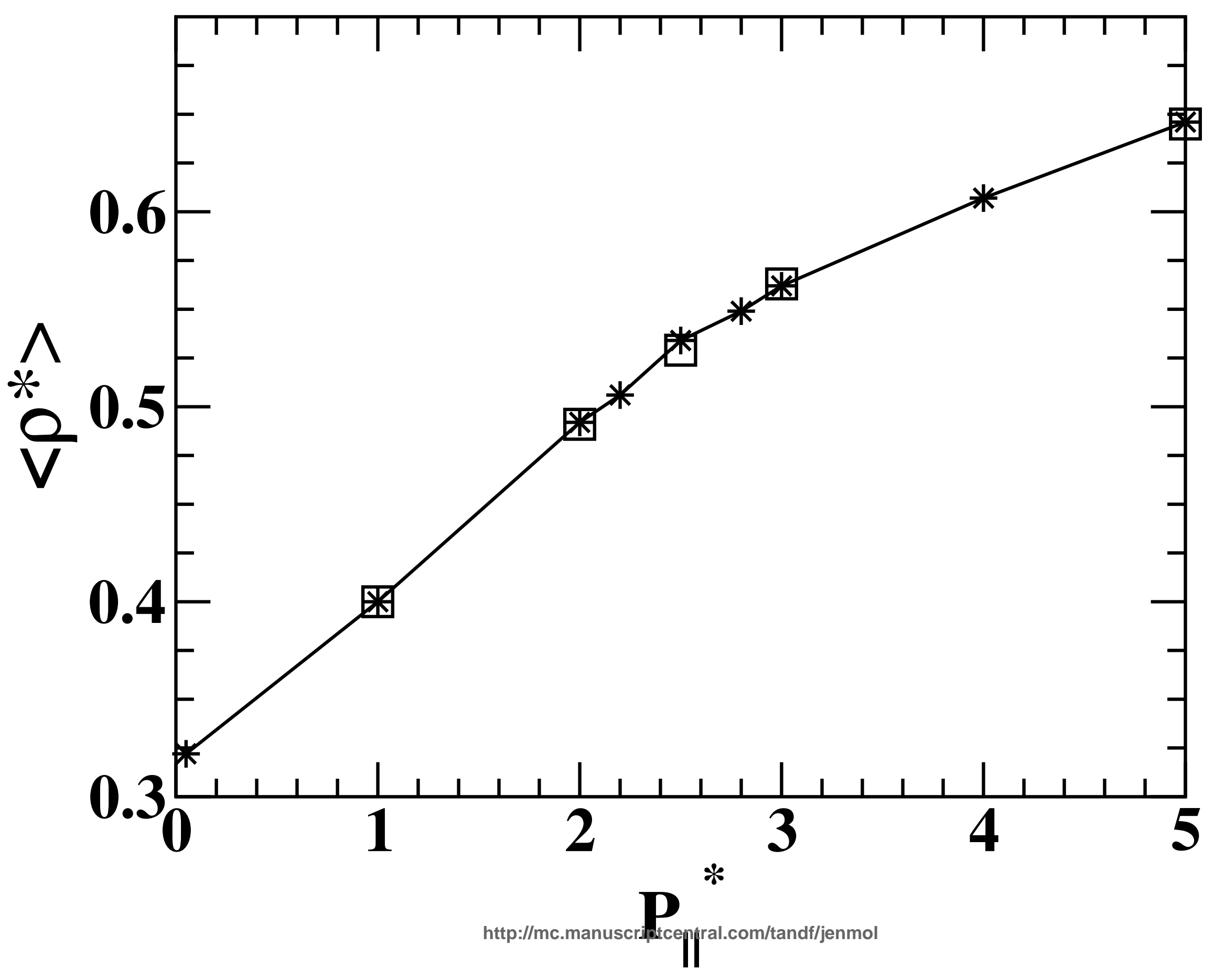




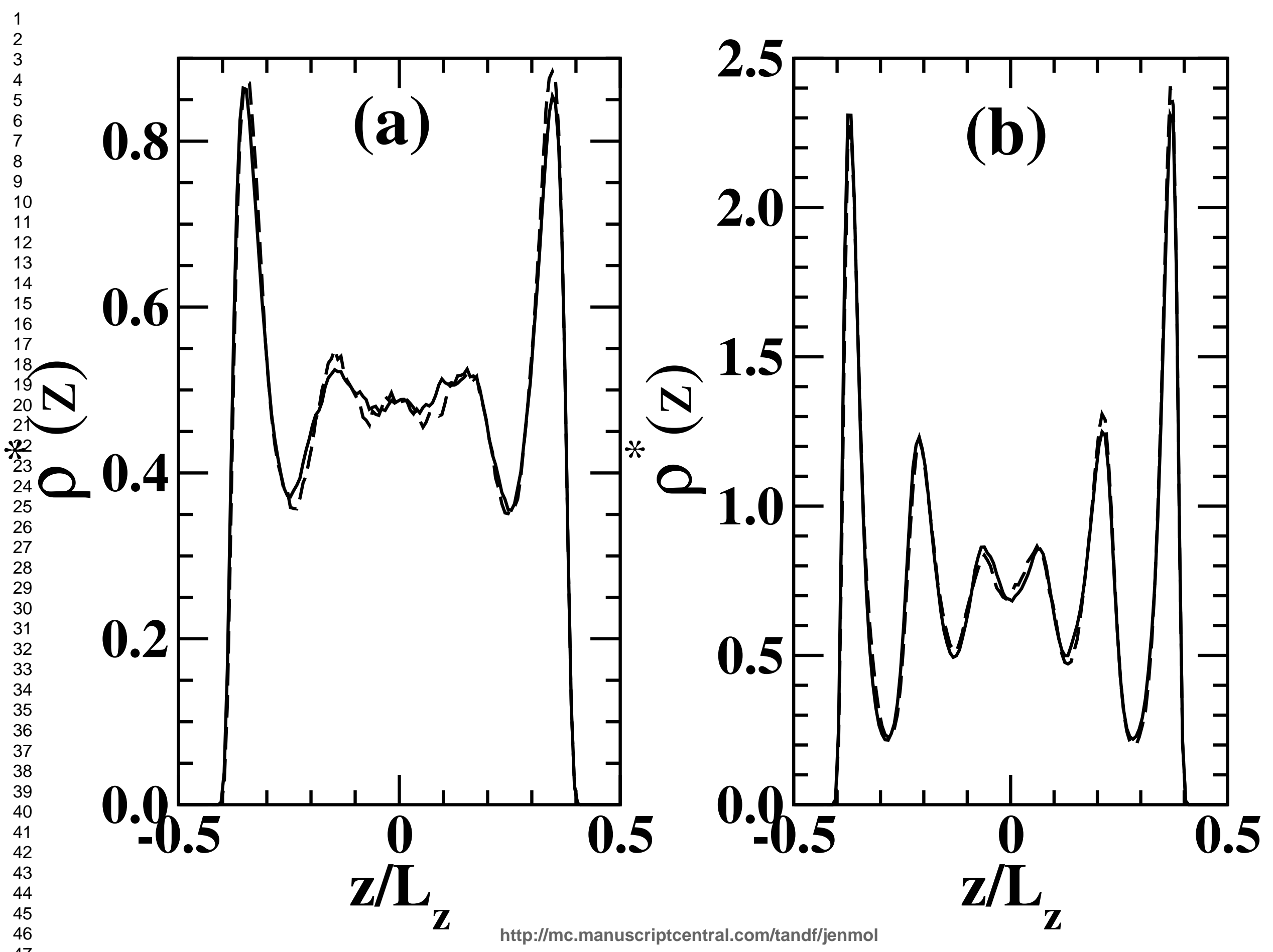




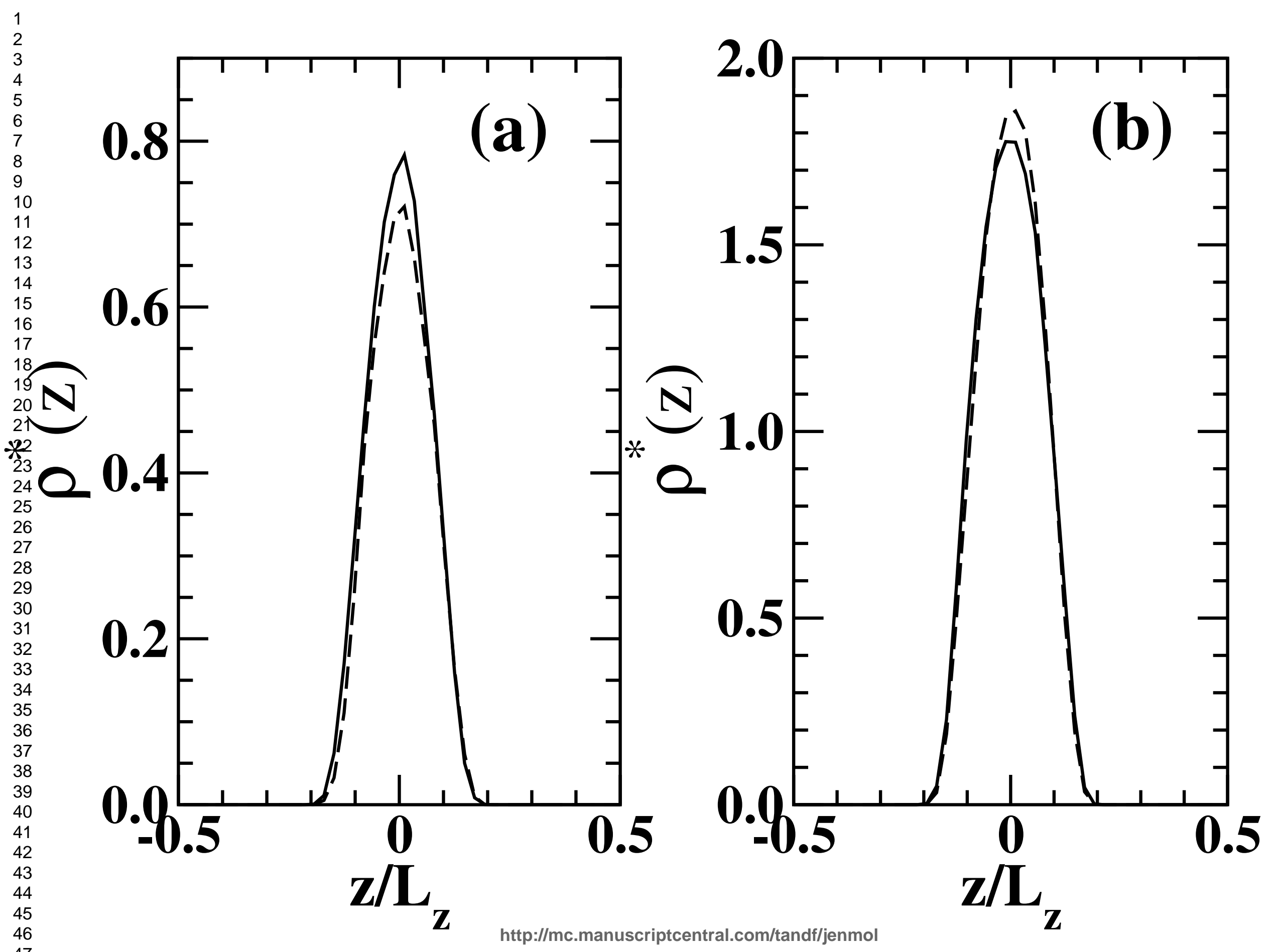




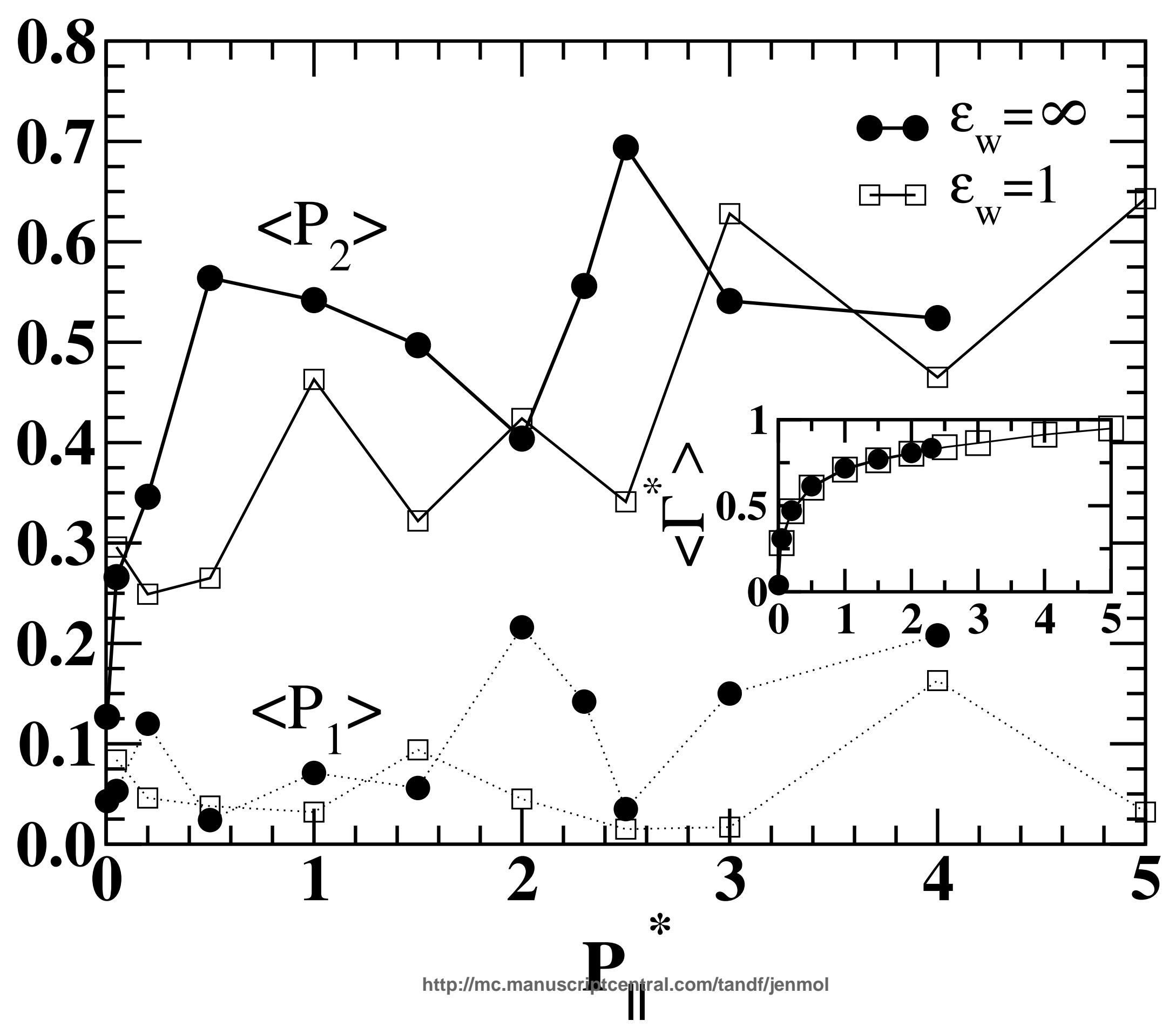




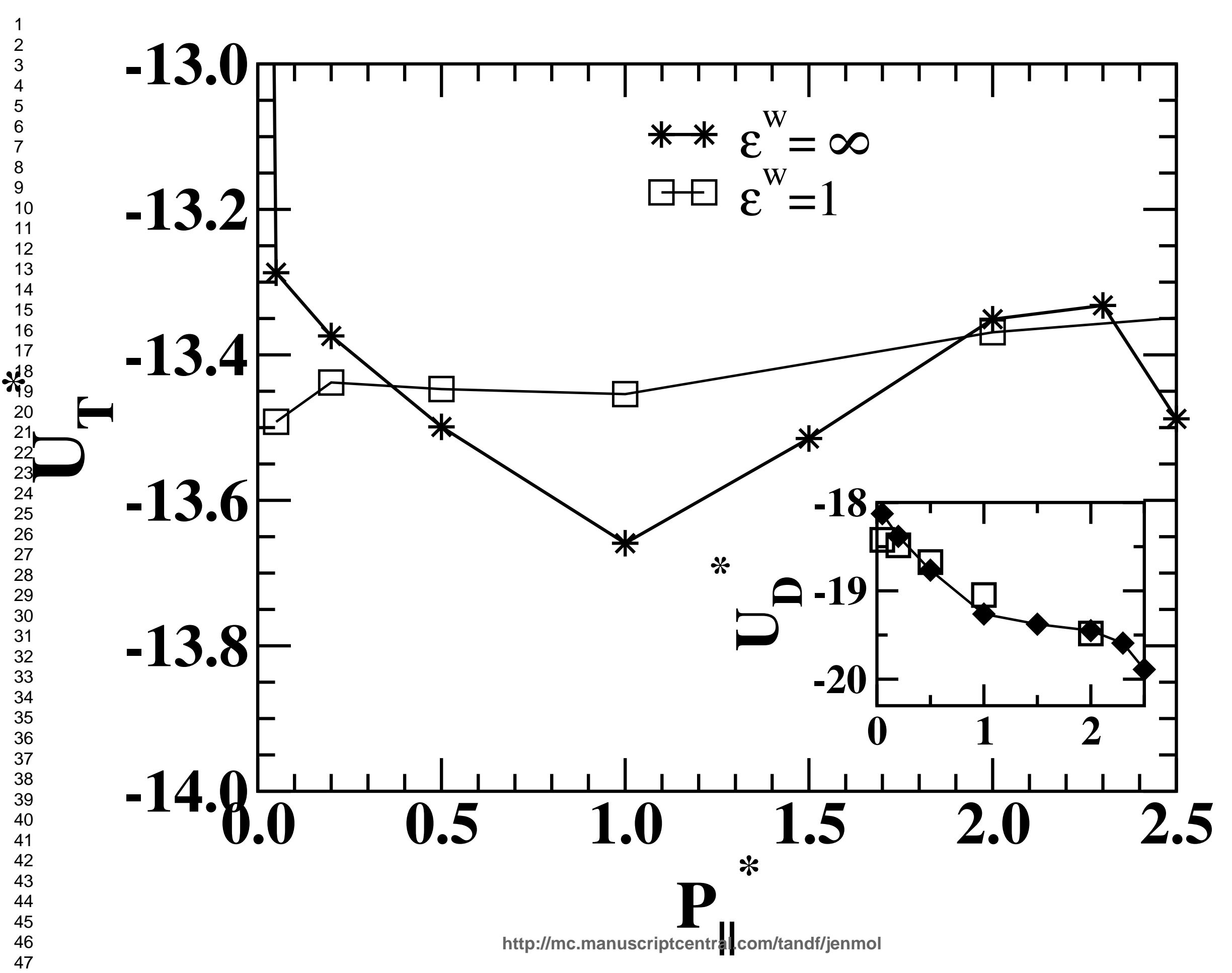




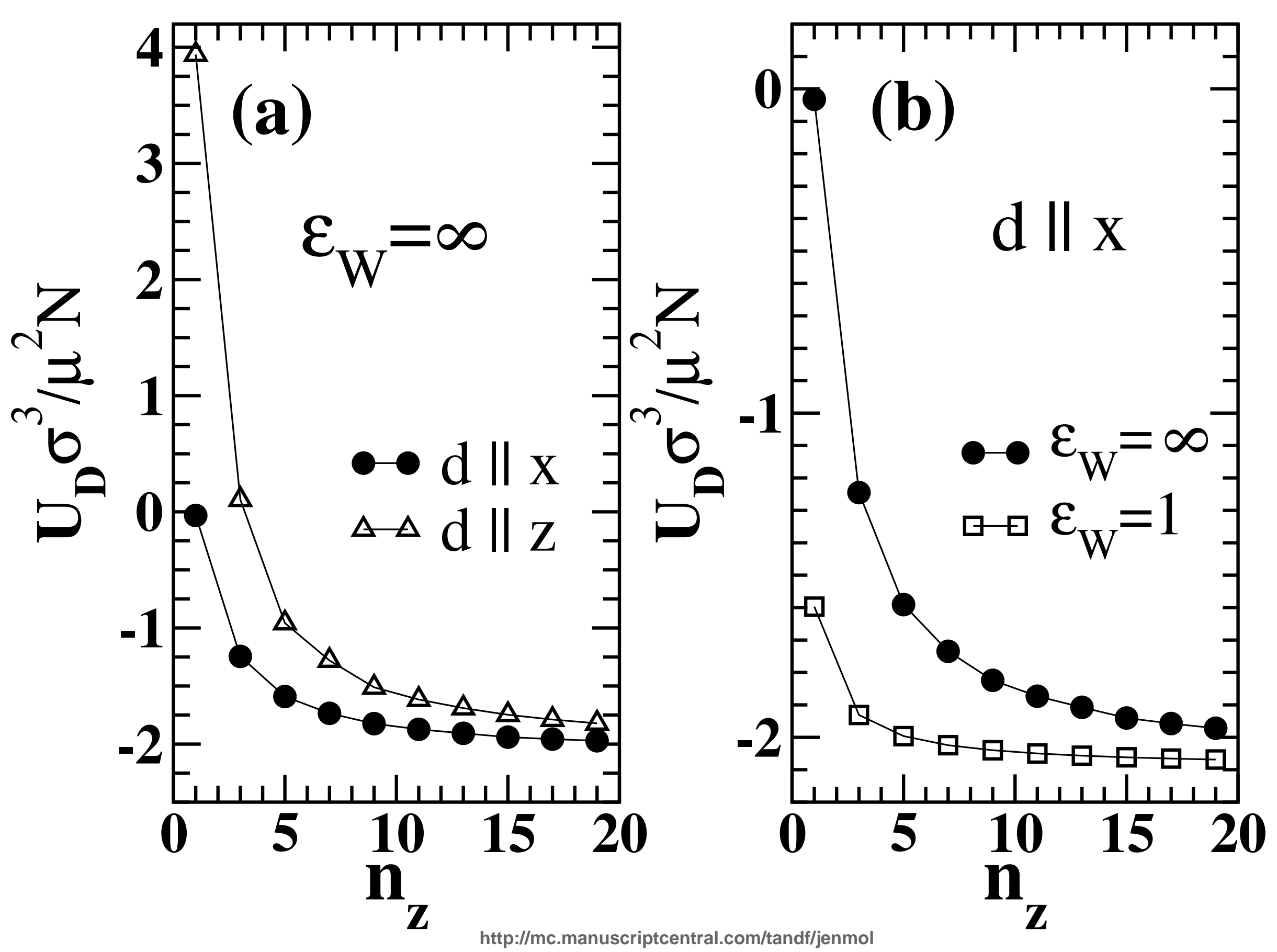

